\title{
Vortices in coupled planes with columnar disorder and bosonic ladders
}

\author{
E. Orignac and T. Giamarchi \\ Laboratoire de Physique des Solides, Université Paris-Sud, Bâtiment 510, 91405 Orsay, France1
}

\begin{abstract}
The phase diagram of two bosonic chains coupled by interchain hopping and interchain boson boson repulsion is investigated by means of bosonization and renormalization group techniques. It comprises two types of charge density waves, a conventional superfluid phase and a superfluid phase made of condensed boson pairs. The effect of a random potential on that system is also derived . Compared with the one chain case, interchain hopping strongly stabilizes the conventional superfluid phase with respect to Anderson localization. By contrast, the superfluid phase made of condensed boson pairs is much more unstable with respect to Anderson localization than the superfluid phase of the one chain system. An exact mapping of the two strongly correlated bosonic chains onto two coupled vortex planes gives us the phase diagram of the latter system without any further calculation. The vortex system exhibits two types of vortex lattice phase(one with entanglement and another one without entanglement), a normal phase and a phase with bound pairs of vortices. The results for Anderson localization of the bosons allow us to study vortex pinning by columnar defects or twin boundaries and obtain the correlation length of the pinned vortex lattice. We show that interplane vortex hopping strongly reduces vortex pinning by columnar defects but not by twin boundaries. We obtain the melting temperature of the entangled solid. We also derive the critical current by modified Larkin-Ovchinnikov arguments and find a very sharp decrease of critical currents when the system moves into the entangled phase in the case of columnar pins. For strong columnar pins, there is a transition into a pinned solid phase with dislocations. We argue that two multicritical points may exist in the phase diagram.
\end{abstract}

\section{INTRODUCTION}

Localization effects in quantum interacting systems remain, even now an extremely challenging problem. For fermionic systems, although interactions are necessary to describe a certain number of experimental situations, the non-interacting case allows for a reasonable starting point in most situations, allowing to deal with the complications due to disorder $\mathrm{B}$. For bosonic systems, the theoretical situation is much less favorable since repulsive interactions have to be included from the very beginning. Despite its theoretical intricacy, 
the problem of disordered bosons is of importance for a variety of experimental situations such as ${ }^{4} \mathrm{He}$ in aerogels or Vycor 3 , granular superconductors 4 , disordered superconducting films 1 Gand disordered Josephson junctions 8 . More recently this problem has also known a renewed interest, both theoretical and experimental, due to the advent of High Temperature superconductors 10 . In these systems, thermal fluctuations can be strong enough to unpin the Abrikosov Lattice from impurities or even melt it 11,12 thus restoring energy dissipation in the system. Finding a way to pin the vortex lattice more efficiently has therefore become a problem of technological importance 3 for applications of HTSC that also arises important questions in the basic physics of vortex systems. Columnar defects parallel to the magnetic field are expected to greatly enhance the pinning of vortices. From the theoretical point of view, it has been shown that the problem of vortex pinning on columnar defects is equivalent to the problem of Anderson localization in a random potential of two dimensional bosons with repulsive interactions 14,15 . In that mapping, superfluidity of the bosonic system corresponds to a vortex liquid phase and restoration of energy dissipation whereas localization corresponds to pinning of vortices and absence of energy dissipation.

The necessity of keeping the repulsive interactions in disordered bosonic systems is due to the fact in the non-interacting limit, the ground state corresponds to all bosons condensed in the lowest energy eigenstate resulting in an unphysical collapse of the system. As a result, one has to start with a non-disordered boson system with repulsive interactions, and consider the effect of randomness. In a two or three dimensional system, this is an extremely hard problem. One dimensional systems allow for a controlled treatment of the interactions therefore improving the accuracy of the subsequent treatment of disorder. It is thus not surprising that the first analytical solution of this problem 16 was found for the case of one-dimensional dirty bosons. A transition between a superfluid phase and a localized phase upon increasing the repulsion was found. The existence of such a transition received numerical confirmation 17 , and was extended to the more experimentally relevant two dimensional situation by beautiful scaling arguments 18 . Since then localization of bosons in two dimension has been confirmed by a variety of numerical techniques 22.

However despite intensive efforts a controlled microscopic theory of such a transition in more than one dimension is still lacking, and one has to resort to various mean field type approximations, the validity of which is difficult to control23. To try to interpolate between the one-dimensional solution and a higher dimensional one, we thus study in this paper the model of a two leg bosonic ladder. Such a system has the advantage of containing interchain tunneling, allowing to mimic higher dimensional systems, while retaining the controlled treatment of the interactions possible in the one dimensional world. One thus expects such a study to give some clue about higher dimensional systems. For fermionic or spin system the study of pure24-26 or disordered 27 29 ladders has been extremely fruitful in that respect. The microscopic results for the ladder can also serve as a test-bed for scaling arguments 18 that are expected to be valid in all space dimension. Indeed, the scaling relations where found to be compatible with the exact exponents 11 found in the single chain superfluid-insulator transition. In addition one expects our study to be directly relevant for experimental systems such as Josephson junction networks that are practical realization of one-dimensional bosons 8 e, but often with more than one channel involved in charge transport.

The ladder situation becomes also specially important when dealing with vortex systems 
in Type II superconductor ${ }^{10}$. The superfluid to insulator transition that occurs in the dirty bosons system when interparticle repulsion is increased results in the equivalent vortex system in a transition from an unpinned vortex liquid to a pinned vortex solid as temperature is lowered. This pinned phase is known as "Bose Glass" phase and its physical properties have been analyzed in Ref. 15 and have been the object of intense experimental studies 30 - 32 .

Treating such system analytically proves to be difficult and possible only in the elastic limit where not dislocations are allowed in the lattice structure 33 . Although it is indeed the case for weak disorder at low temperature, as for point like disorder 36 where the existence of a dislocation free glassy phase was proven both analytically 35.3739 and numerically 40 such a restriction cannot allow to approach the melting transition nor the case of strong disorder where dislocations obviously play a role. It is thus interesting to study special geometry, where dislocations could be treated exactly. This is for example the case for vortices confined into planes parallel to the magnetic field 141 , corresponding to coupled chains of one dimensional bosons. This situation may be experimentally achieved by putting a magnetic field parallel to the $\mathrm{CuO}$ planes in superconductors such as $\mathrm{YBCO}$ or BiSSCO 224 . In the case of point like disorder this geometry allowed to confirm the existence of the Bragg glass phase 37 38, and to study the melting and strong disorder case in a controlled way. However in these approaches tunneling of vortices between planes was not treated. When included 5 it was argued that the periodic potential induced by the layers would still lead to a smectic order of the vortices, with even the possibility of supersolid phases between a crystal at low temperature and the liquid (or smectic) at high temperatures.

The ladder system allows for a detailed microscopic solution of such systems, taking both hopping, interactions and disorder into account. It is thus possible to describe more realistically the possible phases of a two dimensional system, and in particular to take into account such effects as the entanglement of the vortices. Indeed in the vortex language, boson tunneling translates as the ability for bosons to overcome the potential barrier that maintains them in the planes by gaining energy from thermal fluctuations and form "superkinks" $t$. Also, the perturbative renormalization group arguments leading to the supersolid phase also applied to the two planes vortex system. Therefore, the existence of such phase can be tested in the two plane system going beyond perturbation theory.

The plan of the paper is as follows: In section II, we recall the bosonization procedure for bosonic systems. Then, we derive the bosonized Hamiltonian of two coupled bosonic chains. This allows us to obtain the phase diagram of the two chain bosonic system using renormalization group arguments. In section III] we consider the effect of random potential scattering. We obtain the phase diagram in the presence of disorder as well as the boson localization lengths and discuss the physical consequences. In section $\mathbb{\mathbb { V }}$, we consider first the mapping of interacting vortices confined in planes onto interacting bosons. We give a physical interpretation of the quantities defined in the boson language in terms of vortices. We discuss the phase diagram of the vortex system in the absence of columnar disorder. Then, we discuss the effect of columnar disorder and compare with the one chain system. Finally, we give a calculation of the critical current of the coupled planes vortex system. Conclusion can be found in section $\mathrm{\nabla}$. Some technical details have been put in the appendixes. 


\section{PURE BOSON LADDER: PHASE DIAGRAM}

\section{A. Hamiltonian}

Let us consider the most general bosonic ladder with both interchain hopping and interchain interactions. Its Hamiltonian is

$$
\begin{aligned}
H= & \int d x\left[\frac{\nabla \psi_{B 1}^{\dagger} \cdot \nabla \psi_{B 1}}{2 m}+\frac{\nabla \psi_{B 2}^{\dagger} \cdot \nabla \psi_{B 2}}{2 m}\right]+\int d x d y U(x-y) \rho_{B 1}(x) \rho_{B 1}(y) \\
& +\int d x d y U(x-y) \rho_{B 2}(x) \rho_{B 2}(y)+\int V \rho_{B 1}(x) \rho_{B 2}(x) d x \\
& +\int t_{\perp}\left(\psi_{B 1}^{\dagger}(x) \psi_{B 2}(x)+\psi_{B 2}^{\dagger}(x) \psi_{B 1}(x)\right) d x
\end{aligned}
$$

where $t_{\perp}$ is the interchain hopping, $U$ and $V$ are respectively the intrachain and interchain interactions between the bosons. Although we used for (国) a continuous representation it of course also applies to discrete models (such as e.g. the bosonic Hubbard model) provided the boson filling is incommensurate with the lattice (for the case of commensurate filling see Ref. 46 for a single chain or Ref. 29 for a commensurate ladder). Physically, one has $t_{\perp}<0$ and $V>0$. To treat (1) is it convenient to use an alternate boson representation of the original bosonic degrees of freedom 4 . The salient points of this bosonization of bosons can be found in Appendix A where the single boson operator and the density operator are expressed as (see Appendix A)

$$
\begin{aligned}
& \rho_{B}(x)=\left(\rho_{0}+\frac{\partial_{x} \phi}{\pi}\right) \sum_{m=-\infty}^{\infty} e^{2 \imath m\left(\phi(x)+\pi \rho_{0} x\right)} \\
& \psi_{B}(x) \sim e^{\imath \theta(x)}\left[\rho_{B}(x)\right]^{1 / 2} \sim e^{\imath \theta(x)} \sum_{m=-\infty}^{\infty} e^{2 \imath m\left(\phi(x)+\pi \rho_{0} x\right)}
\end{aligned}
$$

where $\rho_{0}$ is the average boson density, $\theta(x)=\pi \int_{-\infty}^{x} d x^{\prime} \Pi\left(x^{\prime}\right)$ and $\phi$ and $\Pi$ are conjugate operators and $a$ is a short distance cutoff. The Hamiltonian (1) is bosonized by treating separately the two chains. In (1), a continuum Hamiltonian is used, but the bosonization procedure would also work for a lattice Hamiltonian. To do so, one introduces the two sets of conjugated fields $\theta_{1}, \phi_{1}$ for chain 1 and $\theta_{2}, \phi_{2}$ for chain two. In the absence of $t_{\perp}$ and $V$ the low energy properties of each chain are described by a Hamiltonian of the form (A1) describing the sound waves which are the phonon modes typical of a Bose superfluid. All the interaction effects are hidden in the so called Luttinger liquid parameters $u$ and $K$ (for each chain). $u$ is the sound wave phase velocity whereas $K$ controls the asymptotic decay of the correlation functions (see appendix A). $u$ and $K$ can be extracted directly out of physical quantities such as compressibility and charge stiffness, as explained in Appendix A, regardless of the precise form of the interaction $U$. For noninteracting bosons $(U \rightarrow 0)$ $K \rightarrow \infty$, whereas a local hard core repulsion would correspond to $K=1$. A longer range interaction allows to reach all positive values of $K$. Taking for simplicity identical chains one has $u_{1}=u_{2}=u$ and $K_{1}=K_{2}=K$. The interchain boson-boson interactions $V$ and interchain hopping terms $t_{\perp}$ are expressed in terms of the fields $\phi_{1}, \theta_{1}, \phi_{2}, \theta_{2}$ using (2). It is convenient to use the symmetric and antisymmetric linear combinations: 


$$
\begin{gathered}
\theta_{s}=\frac{\theta_{1}+\theta_{2}}{\sqrt{2}}, \theta_{a}=\frac{\theta_{1}-\theta_{2}}{\sqrt{2}} \\
\phi_{s}=\frac{\phi_{1}+\phi_{2}}{\sqrt{2}}, \phi_{a}=\frac{\phi_{1}-\phi_{2}}{\sqrt{2}}
\end{gathered}
$$

In this representation symmetric degrees of freedom and antisymmetric ones decouple leading to the following bosonized form for the Hamiltonian (1)

$$
\begin{aligned}
H & =H_{a}+H_{s} \\
H_{s} & =\int \frac{d x}{2 \pi}\left[u_{s} K_{s}\left(\pi \Pi_{s}\right)^{2}+\frac{u_{s}}{K_{s}}\left(\partial_{x} \phi_{s}\right)^{2}\right] \\
H_{a} & =\int \frac{d x}{2 \pi}\left[u_{a} K_{a}\left(\pi \Pi_{a}\right)^{2}+\frac{u_{a}}{K_{a}}\left(\partial_{x} \phi_{a}\right)^{2}\right]+\frac{t_{\perp}}{\pi \alpha} \int d x \cos \left(\sqrt{2} \theta_{a}\right)+\frac{V \alpha}{(\pi \alpha)^{2}} \int d x \cos \left(\sqrt{8} \phi_{a}\right)
\end{aligned}
$$

The Luttinger liquid parameters for the symmetric and antisymmetric modes are given by

$$
\begin{aligned}
& u_{s, a}=u \sqrt{1 \pm \frac{V K}{\pi u}} \\
& K_{s, a}=\frac{K}{\sqrt{1 \pm \frac{V K}{\pi u}}}
\end{aligned}
$$

where the upper sign is for the $s$ mode. $H_{s}$ has the same form as (A1) so that correlation functions of the symmetric degrees of freedom are easily obtained using (A4). On the other hand, the antisymmetric degrees of freedom are more difficult to treat. To do so one can exploit the analogy of $H_{a}$ with the Hamiltonian encountered for coupled spin chain systems 26.28. $H_{a}$ has two different gapped phases. When $V$ is the most relevant field, $\phi_{a}$ acquires a mean value that minimizes the ground state energy (i.e. $\left\langle\phi_{a}\right\rangle=\frac{\pi}{8}$ for $V>0$ ), whereas the correlation functions of the exponentials of $\theta_{a}$ all decay exponentially fast. When $t_{\perp}$ is the most relevant field the situation is reversed: $\theta_{a}$ has a mean value that minimizes the ground state energy (i. e. $\left\langle\theta_{a}\right\rangle=0$ ) and all exponentials of $\phi_{a}$ decay exponentially. In the first case, the system is dominated by interchain repulsion and the density fluctuations between the two chains are frozen. In the second case interchain hopping dominates corresponding to the standard Josephson coupling and assuring that the superconducting phase is identical $\left(\left\langle\theta_{a}\right\rangle=0\right)$ between the two chains. The boundary $K_{a}^{c}$ between these two regimes depends on the values of $V$ and $t_{\perp}$. A way to estimate the boundary is to consider that the most relevant field is the one with the shortest correlation length. Using the standard formula for the correlation length of sine-Gordon time equations (see appendix A) one obtains for the values at the boundary (for small $V, t_{\perp}$ )

$$
\frac{\log (V)}{\log \left(t_{\perp}\right)}=\frac{2-2 K_{a}^{c}}{2-1 / 2 K_{a}^{c}}
$$

For $K=1 / 2$ the transition occurs when $V=t_{\perp}$. Let us not that since $\phi$ and $\theta$ are conjugate fields it is not possible to get simultaneous order in both fields even if at the perturbative level both operators in the Hamiltonian can be relevant simultaneously. The schematic phase diagram is shown on Figure 1 . 


\section{B. Correlation functions and phase diagram}

As a consequence of the existence of the gap in the antisymmetric degrees of freedom, most of the operators have exponentially decaying fluctuations, and the associated susceptibilities remain finite. Some operators however have algebraic correlations and diverging associated susceptibilities. These are the following

$$
\begin{aligned}
O_{S F+} & =\psi_{B 1}+\psi_{B 2}=e^{\imath \frac{\theta_{s}}{\sqrt{2}}} \cos \left(\frac{\theta_{a}}{\sqrt{2}}\right) \\
O_{C D W^{4 \pi \rho_{0}}} & =\rho_{B 1,4 \pi \rho_{0}}+\rho_{B 2,4 \pi \rho_{0}} \sim e^{\imath \sqrt{8} \phi_{s}} \\
O_{C D W-} & =\rho_{B 1,2 \pi \rho_{0}}-\rho_{B 2,2 \pi \rho_{0}}=\frac{e^{\imath \sqrt{2} \phi_{s}}}{2 \pi \alpha} \sin \left(\sqrt{2} \phi_{a}\right) \\
O_{B P S F} & =\psi_{B 1}(x) \psi_{B 2}(x) \sim e^{\imath \sqrt{2} \theta_{s}}
\end{aligned}
$$

The $C D W$ order parameters describe "crystalline" type phases, whereas the $S F$ ones correspond to superfluid phases. The $O_{C D W}$ - operator is the order parameter of an antisymmetric charge density wave. In that phase, there is a charge density wave on each charge of the ladder. These charge density waves have the same $2 \pi \rho_{0}$ wavevector and are dephased so that a minimum of the density on one leg of the ladder corresponds to a maximum of the density on the other leg. Since we consider mainly interchain repulsion $V>0$ we confine ourselves to this order parameter. For an attractive interchain interaction one would have a corresponding $O_{C D W}+$ order parameter (with $\cos \left(\sqrt{2} \phi_{a}\right)$ instead of $\sin \left(\sqrt{2} \phi_{a}\right)$ ) corresponding to a phase for which the two $2 \pi \rho_{0}$ CDW on each chain would be in phase. The $O_{C D W^{4 \pi \rho_{0}}}$ operator is the order parameter of a symmetric charge density wave with $4 \pi \rho_{0}$ wavevector. This corresponds to bosons that form a charge density wave with a period half the one of the CDW-. The physical picture is that of bosons forming a CDW- but hopping from one chain to the other thus minimizing the hopping energy. This has the effect of halving the period and changing an antisymmetric charge density wave into a symmetric one. Strictly speaking, the order parameter for the $4 \pi \rho_{0}$ charge density wave is in fact:

$$
O_{C D W^{4 \pi \rho_{0}}}=e^{\imath \sqrt{8} \phi_{s}} \cos \sqrt{8} \phi_{a}
$$

and expression (10) results from the combination of the term $V \cos \sqrt{8} \phi_{a}$ of equation (5) with equation (13) in the perturbative expansion of the correlation functions. The $O_{S F+}$ operator is the order parameter of a superfluid phase in which there is Bose condensation of the bosons that occupy the bonding band. This is the normal superfluid order parameter. On the other hand the $O_{B P S F}$ operator is the order parameter of a superfluid phase in which pairs of bosons that are formed along the rungs present a Bose condensation. In a lattice system, these pairs would be formed of a boson on $i$-th site of chain 1 , and another boson on the $(i+1)$-th site of chain 2 for a repulsive $V$ (on the same site for $V<0$ ). This implies that the BPSF also contains antisymmetric charge density wave (i. e. CDW-) fluctuations. Which phase is realized depends on whether interchain hopping or interchain interactions dominate. The $\mathrm{CDW}^{4 \pi \rho_{0}}$ and $\mathrm{SF}+$ phases are typical of a system dominated by interchain hopping whereas the CDW- and BPSF phase correspond of a system dominated by interchain repulsion. Using (5) one can obtain the generic phase diagram for the boson 
ladder. The gapped fields, their mean value and the phase that obtains are listed in table [. The resulting phase diagram is shown on Figure 2. For moderate intrachain repulsion $K_{s}>1$, and only the superfluid phase is possible. If $V$ is small with respect to $u / K$, $K_{a}=K+O(K V / u), K_{s}=K+O(K V / u)$ and one moves on a special line of the phase diagram with $K_{a} \sim K_{s}$ (dotted line on Figure 2). Note that, as can be seen from (6), increasing the small $q$ interchain repulsion pushes paradoxically the system to a regime where the interchain hopping is more relevant and thus destabilizes the crystal. This is due to the fact that now regions of high density on one chain face regions of low density on the other making it easier for the hopping to act. Therefore, in a system of two coupled chains of bosons with repulsive local interchain interactions the BPSF does not exist (see Figure 2), which is physically reasonable. Instead, one has a superfluid SF+ phase for $K>1 / 2$ and a CDW- phase for $K<1 / 2$ corresponding to a boson "crystal". For attractive enough interchain interactions one could expect to stabilize the BPSF phase. Finally let us make the connection between the bosonic ladder and other models. First using the standard equivalence between spins and hard core bosons (see e.g.49), the bosonic ladder can be mapped to a spin ladder. In the absence of magnetic field for the spin system, one would have a commensurate filling of one boson every two sites. The massive phases (Haldane or singlet) for the spin system would thus correspond to an insulating Mott phases for the bosonic ladder, where bosons are frozen along the chains but able to hop between the two chains. The Ising antiferromagnetic phase corresponds to the boson "crystal". The massless XY phases are the superconducting ones, the BPSF phase being equivalent to the XY2 phase. Since such systems have been studied in details both for the pure system and in the presence of disorder we refer the reader to Ref. 29 for a more detailed analysis of the physical properties. Here we mainly focus on the incommensurate situation that can be mapped to the spin ladder in the presence of a finite magnetic field, playing the role for the bosonic ladder of the chemical potential. Such bosonic ladders can also be mapped to the standard fermionic ladder in the presence of an on-site attraction 28 . In that case the electrons pair in bound states and single electron hopping becomes irrelevant. The system becomes equivalent to pairs of carriers hopping for site to site with an effective hopping of the order of $t^{2} /|U|$ for a strong attraction $U$. The interchain hopping can also be viewed in that case as the standard Josephson coupling between two superconducting chains. More details can be found in ref. 28.

\section{EFFECT OF DISORDER}

Let us now add disorder to the bosonic ladder. In a continuum system, the only possible form of disorder is a random chemical potential. In the case of a lattice system, another possible form of disorder besides a random on site potential is random hopping along the chains. Although these two types of disorder can lead in principle to different physical phases when particle hole symmetry exists (as is for example the case for spin chains29.50), for an incommensurate density, they lead to the same coupling in the continuum limit. Since we confine our study of the bosonic ladder to such a case, we can restrict ourselves to consider a random chemical potential. The disorder is thus modeled as two uncorrelated random Gaussian potentials in chains 1 and 2. In the continuum limit, the coupling of the bosons 
with those random potentials is:

$$
H_{\text {disorder }}=\int d x\left[V_{1}(x) \rho_{B 1}(x)+V_{2}(x) \rho_{B 2}(x)\right]
$$

where $\overline{V_{p}(x) V_{p^{\prime}}\left(x^{\prime}\right)}=D \delta_{p, p^{\prime}} \delta\left(x-x^{\prime}\right)$. Upon bosonization the full Hamiltonian including the coupling with disorder has the following form

$$
\begin{aligned}
H & =H_{a}+H_{s}+H_{\text {disorder }} \\
H_{\text {disorder }} & =\int d x \sum_{m}\left[V_{1}^{2 m \pi \rho_{0}}(x) e^{\imath m\left(\sqrt{2}\left(\phi_{a}+\phi_{s}\right)(x)\right)}+V_{2}^{2 m \pi \rho_{0}}(x) e^{\imath m\left(\sqrt{2}\left(\phi_{a}-\phi_{s}\right)(x)\right)}+\text { H. c. }\right]
\end{aligned}
$$

where $H_{a}, H_{s}$ are given by (5) and $\overline{V_{p}^{2 m \pi \rho_{0}}(x) V_{p^{\prime}}^{2 m \pi \rho_{0}}\left(x^{\prime}\right)}=D_{m} \delta\left(x-x^{\prime}\right) \delta_{p, p^{\prime}}$. The $q \sim 0$ part of the random potentials has been dropped since it does not affects the gaps for small disorder and does not induce localization as discussed in Ref. 28. As usual the most relevant coupling to disorder is given by the lowest harmonic 16 so we retain for the moment only $V^{2 \pi \rho_{0}}$ and $D_{1} \sim D$. Higher harmonics can be taken into account in a similar way using (2). The analysis of the phase diagram of (15) proceeds in a similar way than for spin ladders 29 , with the important physical difference of a gapless symmetric sector for the boson case. Disorder has different effects on the phases dominated by interchain hopping (i.e. with $\left\langle\theta_{a}\right\rangle=0$ ) corresponding to sectors I and II of table 1 and the ones dominated by interchain repulsion

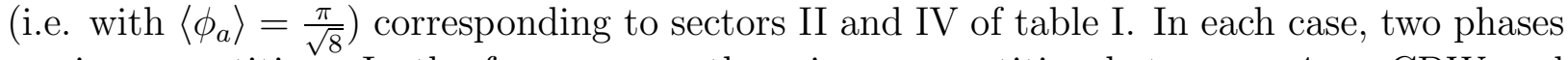
are in competition. In the former case, there is a competition between a $4 \pi \rho_{0} \mathrm{CDW}$ and a $\mathrm{SF}+$ phase. The $4 \pi \rho_{0} \mathrm{CDW}$ phase can gain energy by pinning on the $4 \pi \rho_{0}$ component of the random potential whereas in the SF+ phase, density fluctuations are too small to permit gaining energy from the random potential. In the latter case, the competition is between the $2 \pi \rho_{0}$ charge density wave and the BPSF phase. The mechanism is similar, however one expects that the stabilization of the CDW- is stronger than the stabilization of the $4 \pi \rho_{0}$ CDW. Technically, the case of the competition of the CDW- and the BPSF phases is simpler and is discussed first.

\section{A. System dominated by interchain repulsion}

When the system is dominated by interchain repulsion, the two phases in competition are the $C D W-$ and the BPSF phase. The effective Hamiltonian describing the coupling with disorder is obtained by replacing $\phi_{a}$ by its mean value in (15). It has the form :

$$
H_{\text {effective }}=\int V_{\text {eff. }}(x) e^{\imath \sqrt{2} \phi_{s}}
$$

where $V_{\text {eff. }}(x)=\left|\left\langle e^{\imath \sqrt{2} \phi_{a}}\right\rangle\right| \imath\left(V_{1}(x)-V_{2}(x)\right)$ and only the most relevant (in the RG sense) terms have been kept. From (16) the renormalization group equation for disorder is straightforwardly obtained in the form:

$$
\frac{d D}{d l}=\left(3-K_{s}\right) D
$$


The renormalization group equation for $K$ is :

$$
\frac{d K}{d l}=-C^{2} D
$$

where $C$ is a non-universal prefactor. In a similar way than for a single chain 16 these RG equations have a fixed line on which disorder is irrelevant, and a strong coupling disordered fixed point. For small $D$, the renormalization of $K$ can be neglected and (17) is sufficient to determine whether the system flows to the fixed line or to the strong coupling fixed point. According to (17), the disorder is relevant for $K_{s}<3$ and irrelevant for $K_{s}>3$. The strong coupling fixed point is in this case an antisymmetric charge density wave CDWpinned on the $2 \pi \rho_{0}$ component of disorder 16.28. Therefore, the BPSF phase is unstable with respect to a very small amount of disorder unless $K_{s}>3$. This is due to the coupling of the random potential to the slowly decaying CDW- fluctuations (see equation (11)) that are subdominant in the BPSF phase. When interchain density-density interactions dominate the superfluid-insulator transition occurs thus for much less repulsive interactions $(K=3)$ than for the single chain case $(K=3 / 2)$. The situation is reminiscent of what happens in fermionic ladders 28 , and is due to the reduction of quantum fluctuations by the freezing of the transverse degrees of freedom. The localization length of the bosons in the pinned phase is easily extracted from the RG equation as the length for which the renormalized disorder is of order one. It gives

$$
l_{\text {loc. }, 2 \text { ch. }} \sim\left(\frac{1}{D}\right)^{\frac{1}{3-K_{s}}}
$$

and as can be expected is much shorter than the localization length of a single chain

$$
l_{\text {loc., } 1 \text { ch. }} \sim\left(\frac{1}{D}\right)^{\frac{1}{3-2 K_{s}}}
$$

Note that as for the fermion case 28 the change in localization length occurs through a change in exponent and not as a simple multiplicative factor. Since the Bose fluid that condenses is made of bosonic pairs, to obtain the exponent of the correlation function of the superfluid order parameter at the transition one must consider

$$
\left\langle T_{\tau} O_{B P S F}^{\dagger}(x, \tau) O_{B P S F}(0,0)\right\rangle=\left(\frac{1}{x^{2}+(u \tau)^{2}}\right)^{\eta_{c} / 2}
$$

as the superfluid order parameter correlation function. That correlation function decays as $r^{-1 / K_{s}}$ in the superfluid phase and at the transition point, $K_{s}=3$. Thus, at the transition point $\eta_{c}=1 / 3$. It is interesting to remark that the same exponent at the transition point obtains in the case of one bosonic chain 1 . Using the RG one can also compute the conductivity in the case of a charged Bose fluid made for instance of Cooper pairs. This gives an indication of the amount of dissipation that is generated in the system upon introduction of disorder. In a neutral boson system, conductivity measures the normal current that would be induced by a gradient in chemical potential. The high frequency dependence of the a.c. conductivity can be obtained in the form : 


$$
\sigma_{\text {a. c. }}(\omega)=\omega^{K_{s}-4}
$$

using the methods exposed in Refs. 16,28. From the a. c. conductivity, it is easy to deduce the temperature dependence of the d. c. conductivity. In the calculation of Refs. [16]28, frequency just serves in determining a cut-off length $\frac{u_{s}}{\omega}$. Similarly, temperature provides a cutoff length $\frac{\hbar u_{s}}{k_{B} T}$. This leads to the following expression for the temperature dependence of the d. c. conductivity :

$$
\sigma_{\text {d.c. }}(T)=T^{2-K_{s}}
$$

For $T \ll T_{\text {pin. }}=\frac{u_{s}}{l_{2 \text { ch. }}}$ or $\omega \ll T_{\text {pin. }}$, the conductivity goes to zero exponentially fast.

\section{B. System dominated by interchain hopping}

In a system dominated by interchain hopping, the two phases in competition are the $\mathrm{SF}+$ phase and the $\mathrm{CDW}^{4 \pi \rho_{0}}$. Since in a system dominated by interchain hopping, $\left\langle\theta_{a}\right\rangle=0$, the massive degrees of freedom in (15) must be integrated out in order to have a non trivial effective coupling to disorder. Alternatively one can couple to a higher harmonic of the random potential. Using the method of Ref. 28, the effective coupling to disorder gives

$$
H_{\text {imp, eff. }}=\int d x V_{\text {eff. }}(x) e^{i \sqrt{8} \phi_{s}}+\text { H. c. }
$$

where $\overline{V_{\text {eff. }}(x) V_{\text {eff. }}\left(x^{\prime}\right)}=C_{1} D^{2} \delta\left(x-x^{\prime}\right) . C_{1}$ is a constant that comes from the integration of the gapped modes 28 . Physically, this represents the coupling of the impurity potential with the $4 \pi \rho_{0}$ component of the density (see $(\sqrt{10})$ ). Note that the genuine $4 \pi \rho_{0}$ component of the random potential is coupled to a $\cos \left(\sqrt{8} \phi_{a}\right)$, so that its contribution is a second order term $V^{2} D_{2}$. In this section we will thus use $\bar{D}$ for the effective disorder. The RG equation for disorder is obtained from (24) as

$$
\frac{d \bar{D}}{d l}=\left(3-4 K_{s}\right) \bar{D}
$$

The impurity potential is relevant for $K_{s}<\frac{3}{4}$ and irrelevant for $K_{s}>\frac{3}{4}$. Therefore, the $\mathrm{SF}+$ phase is stable with respect to Anderson localization for $K_{s}>\frac{3}{4}$, corresponding to extremely repulsive interactions (this would need hard core bosons plus a nearest neighbor repulsion). For $K_{s}<\frac{3}{4}$, the randomness is relevant and the $\mathrm{SF}+$ phase is replaced by a $4 \pi \rho_{0}$ CDW pinned on the disorder. The pinning length of the CDW, which is also the localization length of the bosons, is given by :

$$
l_{\text {loc. }, 2 \text { ch. }} \sim\left(\frac{1}{\bar{D}}\right)^{\frac{1}{3-4 K_{s}}}
$$

This time, the localization length is much larger than the localization length of the one chain system (20), indicating that the two chain system is less localized than its one chain counterpart. In particular, a system of two hard-core bosons chains (which has $K=1$ ) with interchain hopping would be superfluid even in the presence of a weak random potential, 
whereas its one chain counterpart would have been already localized $\left(K_{c}=3 / 2\right)$. The critical exponent of the superfluid order parameter correlation function is obtained by the method of the preceding section. In the superfluid phase, the 2 point correlation function of $S F+$ decay as $r^{-1 / 4 K_{s}}$. Since the phase boundary is at $K_{s}=3 / 4$, the exponent of the superfluid order parameter at the transition point is $1 / 3$ as in the preceding section. The high frequency dependence of the conductivity is computed as:

$$
\sigma_{\text {a.c. }}(\omega)=\omega^{4 K_{s}-4}
$$

using the methods of Refs. 16,28. Due to the equivalence of frequency and temperature one also has:

$$
\sigma_{\text {d.c. }}(T)=T^{2-4 K_{s}}
$$

As in the preceding section, high frequency conductivity refers to a.c. conductivity at frequency above the characteristic pinning frequency $u_{s} / l_{2 \mathrm{ch}}$. The full phase diagram in the presence of disorder is represented on Fig. 3

\section{Physical discussion}

Although most of the physical realization of bosonic ladders are likely to come from classical systems (see next section), its study also present direct interest for quantum systems. Indeed recently practical realization of one dimensional bosonic systems have been obtained, mostly based on Josephson junction arrays 9 . It would be interesting to check in these systems various of the predictions made here. The easiest to determine is certainly the strong stabilization of the superfluid phase for the ladder in comparison with the single chain case. For the pure system, for a commensurability of one boson per site the Mott

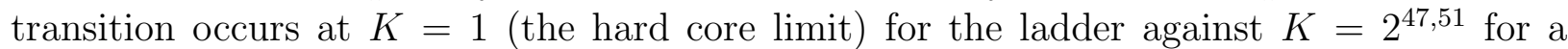
single chain, i.e. much less repulsive interactions. In the presence of disorder a similar stabilization occurs since the transition goes from $K=3 / 2$ for a single chain to $K=3 / 4$ for the ladder. Maybe even easier to investigate would be the bosonic ladder in the presence of a single barrier. The properties of uniform disorder and a single impurity 5253 can be easily connected 5 which allows from the present study to get the parameters for which the barrier would remain transparent. For a single bosonic chain the barrier is not effective for $K>1$ whereas for the ladder the limit would be $K>1 / 2$. Another, albeit more theoretical interest of the bosonic ladder is to provides another analytic system on which the general scaling arguments 18 proposed for the superfluid-insulator transition can be checked. Indeed at that time these arguments could only be benchmarked on the only existing analytical solution for a superfluid-disordered transition, namely the $d=1$ single chain case 1 . Although since then these scaling predictions have been found in agreement with various numerical simulation 20.1921. it is still interesting to verify them on models for which the exponents can be computed exactly. The equality $z=d$ for the dynamical exponent clearly still holds for the ladder since the system is still described by a Luttinger Liquid. Similarly it is easy to check from the RG equations that the compressibility still remains finite at the transition, again in agreement with the scaling prediction. Finally,in Ref. [18, a bound for the exponent 
of the superfluid order parameter at the transition point has been derived by combining an exact inequality with scaling. When specifying to $z=d=1$, that bound is $\eta_{c} \leq 1$. Since $\eta_{c}=1 / 3$ at the transition, both in the case of the BPSF to insulator transition and $\mathrm{SF}+$ to insulator transition, the bound is indeed verified for the ladder. It would clearly be interesting to extend such an analysis to ladders with more than two legs to complete the check. Finally the two leg ladder prompts for the interesting question of the existence of a universal exponent for the superfluid transition in one dimension $\eta=1 / 3$, regardless of the number of legs. Although a detailed check of this statement would need a complete solution of the models with more than two legs, simple arguments given in Appendix B suggest that this is indeed the case at least in systems in which all modes but the symmetric one are gapped. This is the case of all systems with an even number of legs. However, this lets the possibility that the exponent could be different from $1 / 3$ in systems with an odd number of chains. An interesting question is whether the exponent is also $1 / 3$ for the transition from the pinned charge density wave that occurs along the line $3 / 4<K_{s}<3, K_{a}=1 / 2$ or if it depends of $K_{s}$. It is difficult to answer this question in the framework of the approximation of a disorder much smaller than the gaps, since such approximation breaks down precisely in the vicinity of that line.

Let us note that of course the universality of the exponent holds here for the case where the disorder is much weaker than all the gaps in the system. The argument cannot be applied directly for a two dimensional system.

\section{COUPLED VORTEX PLANES PROBLEM}

Although specially engineered quantum systems are necessary to have coupled bosonic chains, such systems have a natural realization in the classical world of vortices. Indeed, using a standard mapping 14 , 5 space time trajectories of bosonic particles can be mapped onto a problem of interacting flux lines. The disorder in the quantum problem is time (i.e. $z$ ) independent, corresponding in the vortex problem to columnar defects. Defects of that type can be artificially produced by irradiation. In the presence of such defects the vortex system is known to give rise to a "Bose glass" phase corresponding to the localized phase of two dimensional bosons. On the other hand a very interesting situation occurs in a layered system if the magnetic field is aligned along the planes direction. In that case it has been proposed 45 that the additional periodic potential due to the planes can confine the vortices and lead to a smectic like state. If the confinement is strong enough one can view the vortices as existing only in planes with repulsive interactions between adjacent planes. Such coupled planes models are especially interesting to study in their own since they allow a detailed description of the melting transition for the pure system as well as a study of the relevance

of dislocations when disorder is present 37, 38, 41 . However, in most of the studies of such systems hopping of the vortices between planes was neglected. When hopping is included, it was proposed 45 for the pure system that three phases would exist: a solid phase where no hopping is present and the system crystallize due to interplane repulsion, an intermediate supersolid phase where both the interchain repulsion and the hopping are relevant, and a high temperature liquid phase. Since the model does not allow for vortices to exist between the planes the liquid phase also possesses a smectic order along the $z$ direction. For an 
infinite number of planes such phases can only be determined by looking at the relevance of the various operators (interchain repulsion and interchain hopping). For two coupled vortex planes we can use the results of the previous sections to give precise answers to the nature of the various phases. Since the perturbative study of two planes and an infinite number of planes are identical we expect the results to be also of relevance for the more general case. In addition one can study the effect of columnar defects of twin boundary disorder on the phases of the pure system. The more delicate case of point like disorder will be considered elsewhere. The particular case of two coupled planes of vortices could also be of relevance to artificial layered structures where superconducting structures can be separated by arbitrary heights of insulating material 12 - 4 .

\section{A. Mapping of the interacting vortices problem onto a bosonic problem}

\section{Path integral reformulation of the interacting vortices problem in terms of interacting bosons}

In this section, we consider a bilayered superconductor in a magnetic field $H>H_{c_{1}}$ parallel to the bilayers. We make the approximation that the bilayers are sufficiently far apart so that the Josephson coupling of the bilayers can be neglected. We suppose that the bilayers exert a strong enough attraction on the vortices so that they remain confined in the planes. We also suppose that there are columnar defects parallel to the planes and parallel to the magnetic field as shown on Fig. . S. Such system may be realized with copper oxide superconductors with coupled planes (such as BiSSCO) or by epitaxial growth of artificial structures 24 with layers of two superconducting materials, one of them being more efficient in confining vortices.

In the following, we design by $O z$ the direction of the columns and of the magnetic field, by $O y$ the direction perpendicular to the plane and $O x$ the direction such that $(O x, O y, O z)$ forms an orthonormal basis. The position of a point on a flux line $i$ of coordinate $z$ along the $O z$ axis is given by $\mathbf{r}_{i}(z)=\left(r_{i x}(z), r_{i y}(z)\right)$. The Hamiltonian for that system is then:

$H=\int_{0}^{\beta} d z\left[\sum_{i}\left(\frac{\partial \mathbf{r}_{i}}{\partial z}\right)^{2}(z)+\frac{1}{2} \sum_{i \neq j} U\left(\mathbf{r}_{i}(z)-\mathbf{r}_{j}(z)\right)+\sum_{i}\left(V\left(r_{i y}\right)+V\left(r_{i y}-2 a\right)\right)+\sum_{i} v\left(\mathbf{r}_{i}(z)\right)\right]$

$\beta$ being the height of the planes, $2 a$ their distance, $U$ being the vortex-vortex repulsive potential, $V$ the vortex plane interaction and $v$ being the random potential induced by the columnar defects. We consider $v$ to be small, corresponding to weak columnar disorder parallel to the applied magnetic field. Although experimentally such a situation is more difficult to achieve than strong pinning, it allows for an analytic treatment of the problem and at least give a qualitative understanding of collective pinning in such systems. This collective pinning may be probed in high field experiments.

The partition function of that system

$$
Z=\int \mathcal{D} \mathbf{r}_{i}(z) \exp \left(-\frac{H}{T}\right)
$$


is identical14 15 to the one of a system of interacting bosons at temperature $\beta$ with a Planck's constant T. Assuming that the attractive potential imposed on the vortices by the planes is strong enough, the tight binding approximation can be used to simplify the boson Hamiltonian 15. The calculations can be found in appendix $\mathrm{Q}$. The resulting Hamiltonian is identical to (1), i.e. two bosonic chains, with interchain and intrachain boson-boson interactions, interchain hopping and a random intrachain potential. Therefore, we only have to translate the results already derived for the bosonic system in section $\Pi \mathrm{A}$ in the vortex language.

\section{Interpretation of the bosonized vortex Hamiltonian in terms of vortices}

In the case of a single chain, the field $\phi(x)$ has a simple interpretation in terms of flux lines. If $u(x)$ represent the displacements of the vortex lattice in the elastic theory, one has: $\phi(x)=-\pi \rho_{0} u(x)$ by identifying the expressions for the $q \sim 0$ component of the density. As a consequence, the action of the bosonized theory identifies with the elastic Hamiltonian of the vortex lattice. The compressibility $\chi$ and the charge stiffness $\mathcal{D}$ of the bosonic system defined respectively in equations Eqs. (A5) and (A7) have also a simple physical interpretation in terms of vortices. $\chi^{-1}$ is proportional to the bulk modulus of the vortex system. $\chi^{-1}$ is also proportional to the magnetic susceptibility of the system since the vortex number is proportional to the magnetic field. $1 / \mathcal{D}$ is proportional the shear modulus of the vortex lattice. This results from writing the action of the bosonized theory (which is also the Hamiltonian for the vortex lattice elastic theory) and considering the effect of making $\partial_{z} \phi \rightarrow \partial_{z} \phi+\lambda$ on the energy. A detailed derivation can be found in appendix $\mathbb{E}$. In the vortex language, the expression (A3) has been derived directly in Ref. 35 starting from the elastic theory of the one dimensional vortex lattice. However, in the case of a two plane system, the mapping on bosons simplifies the description of vortex tunneling.

\section{B. Phase diagram of the vortex system}

\section{Flux lines in the absence of disorder:}

In the absence of disorder, the vortex system is equivalent to the bosonic system in the absence of random potential discussed in section ПA. The parameter $K_{s}$ controlling the stability of the different phases depends on temperature, vortex density and elastic constants of type vortex lattice as shown in Appendix $\mathbb{E}$. Its expression is:

$$
K_{s}=\frac{\pi \rho_{0}^{2} T}{\left(c_{44} c_{1}\right)^{1 / 2}}
$$

Each of the four bosonic phases translates into a phase of the vortex system. In terms of flux lines, the CDW- is a crystal phase with a periodic array of flux lines in each plane. The periodic array of vortices in the second plane is translated of half a period along the $\mathrm{x}$ axis with respect to the one that lies in the first plane in order to minimize the repulsion energy. In that phase, there is no interplane hopping of vortices. The CDW-is a strict $1 \mathrm{~d}$ analog of a vortex lattice. 
By contrast, the $\mathrm{CDW}^{4 \pi \rho_{0}}$ has the same disposition of vortices but they are constantly hopping from one plane to the other leading to an effective period of the vortex lattice that is half the one of the CDW-. This is a vortex lattice with "superkinks" from one plane to the other. Due to the hopping between the planes this phase is the equivalent in this restricted geometry of an entangled solid phase.

The $\mathrm{SF}+$ is a vortex liquid phase in which a flux line is making "superkinks" from one plane to the other. In this phase, vortices are unpinned. This implies strong dissipation in the original vortex problem (i.e. in the vortex system superconductivity is lost). Since in this phase hopping is relevant vortex entanglement is expected. In other words, the SF+ phase maps onto an entangled liquid.

On the other hand, the BPSF phase is a vortex liquid in which flux lines that are in different planes are bound to each other. No hopping between planes takes place.

As usual in a two dimensional classical system, these phases do not have true long range order but algebraically divergent correlations. It is generally expected that by coupling weakly a lot of two chains systems, the phase that would exist would be the one with the most divergent fluctuations in the isolated two chain system as suggested by mean field theory. In the case of a coupled chain system, there would be true superfluidity of the boson system (leading to an exponential decay of density fluctuations i.e. a true vortex liquid) or to formation of a vortex lattice with true long range order.

In the case: $V(y)=-V_{0} \delta(y)$ and $U(\mathbf{r})=U_{0} \delta(\mathbf{r}), t_{\perp}$ is much larger than $V$, so that $K_{s} \simeq K_{a} \simeq K$. This case corresponds to the dotted line on Fig. 2.

As a consequence, this system has 2 phases: a CDW- for $K_{s}<1 / 2$, and the SF + phase for $K_{m}>1 / 2$. We assume here $V \sim t_{\perp}$ for simplicity otherwise the boundary is displaced according to Figure 1. The behavior of vortices in these two phases is represented on figure 5. In terms of vortices, there is a critical temperature (see appendix E) $T_{m}=\frac{\left(c_{44} c_{1}\right)^{1 / 2}}{2 \pi \rho_{0}^{2}}$ such that for $T<T_{m}$ the vortex system has strong fluctuations towards the vortex lattice state, and for $T>T_{m}$ strong fluctuations towards the vortex liquid state as shown on figure 6 .

When vortex density (i.e. applied magnetic field) is increased, the "melting temperature" $T_{m}$ decreases. Also, the larger the elastic constants, the higher the melting temperature. Such results are very similar to the higher dimensional phase diagram.

Note that since the $\phi$ and $\theta$ fields are conjugated an intermediate "supersolid" phase where one would have both a crystalline and a superfluid order, cannot exist in this system, even if perturbatively both operators would be relevant for $1 / 4<K<1$. A more detailed proof is given for the special temperature corresponding to $K=1 / 2$ in Appendix $\mathbb{F}$. Whether such supersolid phase can exist for an infinite number of planes as advocated in ref. 45 remains an open question.

\section{Effect of columnar disorder on the vortex system}

In the presence of columnar disorder, the vortex system at finite temperature is equivalent to the two chain boson system in the presence of a random potential discussed in section III.

If the interplane interactions are not too strong $K_{s} \sim K$ and the various phases can be read from figure 3 . The pinning of vortices is then controlled by the parameter $K_{s}=\frac{T}{2 T_{m}}$, 
where $T_{m}$ is the melting temperature.

The main effect is to replace the solid-liquid transition that existed in the pure system at $T=T_{m}$ by a double transition. The solid phase is replaced by a pined solid corresponding to the $2 \pi \rho_{0}$ vortex lattice phase. This phase is a Bose glass. At the pure melting temperature $T_{m}$, the solid phase disappears and interplane interactions become irrelevant. The Bose glass phase is replaced by a pinned $\mathrm{CDW}^{4 \pi \rho_{0}}$ vortex glass phase. Since this phase has dominant symmetric density fluctuations is possesses some (disordered) solid type order. On the other hand interplane hopping being relevant, this phase could also be viewed as a pinned solid with entanglement, with intermediate properties between a solid and a liquid. It is only above a higher temperature $T_{L}=3 / 2 T_{m}$ that this entangled solid disappear to give place to the genuine liquid, where disorder is irrelevant, as shown on figure 7. As is expected in the presence of columnar disorder 5 the melting temperature is renormalized upward.

In the two plane vortex system, the pinning of the $2 \pi \rho_{0}$ vortex lattice is much stronger than the pinning of the $4 \pi \rho_{0}$ vortex lattice, as can be shown by the calculation of the pinning lengths and critical currents detailed in the following section. The reason is that the $4 \pi \rho_{0}$ vortex lattice is destabilized by formation of superkinks that permit to average the disorder and reduce its efficiency in pinning the vortex system. This superkink formation is possible only when temperature is large enough to overcome the deformation energy that is needed to bend the vortices, i.e. $T>T_{m}$. In the $2 \pi \rho_{0}$ vortex lattice on the other hand, there is an enhancement of pinning due to strong vortex repulsion that makes the vortex system much more sensitive to the antisymmetric component of the random potential. For finite disorder since the pinning of the $2 \pi \rho_{0}$ vortex lattice is much stronger than the one of the $4 \pi \rho_{0}$ vortex lattice the transition line will be shifted upward from $K_{a}=1 / 2$, i.e. to temperatures higher than $T_{m}$. Columnar defects obviously tend to make hopping between planes less relevant. However, we are in the limit where hopping dominates over columnar disorder, so that the difference in columnar pinning potential between the planes is simply averaged out. Such effect appears nevertheless in renormalization group treatment around the decoupled chains fixed point.

It is interesting to note that if the disorder is perfectly correlated between the planes, as for example would be the case for twin boundaries, it can only couple to the $4 \pi \rho_{0}$ component of the density in both the $2 \pi \rho_{0}$ vortex lattice and the $4 \pi \rho_{0}$ vortex lattice, leading to the same pinning lengths in the two phases up to prefactors. This is easily seen by putting $V_{1}=V_{2}$ in Eq. (15). Such result is due to the fact that the disorder being the same in the two planes, it does not couple to the antisymmetric density of the vortex solid phase, and the averaging effect of vortex hopping cannot lead any more to a reduction of vortex pinning.

As we have seen above, the smaller $\frac{T}{T_{m}}$ the stronger the pinning. Thus, at a given temperature, a stiff vortex lattice having a higher $T_{m}$ is much more easily pinned than a softer one. Also, by reducing vortex density (i.e. reducing the applied magnetic field) one increases $T_{m}$ and thus the pinning of the vortex lattice.

\section{Physical discussion}




\section{Weak disorder limit}

The two planes vortex system shows two superconducting phases with different vortex pinning lengths. The first one, the $2 \pi \rho_{0}$ vortex solid, is dominated by interplane vortex repulsion and has the shortest vortex pinning length : $l_{2 \pi \rho_{0}} \sim\left(\frac{1}{D}\right)^{\frac{1}{3-K_{s}}}$ (see Eq. (20)).

Expressed in terms of temperature, this gives $l_{2 \pi \rho_{0}} \sim\left(\frac{1}{D}\right)^{\frac{T_{1}}{3\left(T_{1}-T\right)}}$, where $T_{1}=6 T_{m}$. It is a Bose Glass phase, and at the melting point, the vortex pinning length becomes infinite with an essential singularity. Such singularity is typical of a two dimensional system. The other superconducting phase, the $2 \pi \rho_{0}$ phase is dominated by formation of superkinks and has a larger pinning length given by Eq. (26) $l_{4 \pi \rho_{0}} \sim\left(\frac{1}{\bar{D}}\right)^{\frac{1}{3-4 K_{s}}}$ since the pinning potential is averaged out on the planes. In terms of temperature, the behavior of the pinning length in this second phase is $l_{4 \pi \rho_{0}} \sim\left(\frac{1}{\bar{D}}\right)^{\frac{T_{L}}{3\left(T_{L}-T\right)}}$, where $T_{L}=\frac{3}{2} T_{m}$. It is again a Bose Glass phase, but the underlying lattice has half the period of the underlying lattice of the previous phase. Similarly to the previous phase, the correlation length shows an essential singularity at the melting point. These two phases could be probed in neutron scattering experiments, and could be distinguished by measuring the wavevector of the underlying lattice.

A quantity of interest for vortices is of course the critical current. A proper calculation of the critical current involves a full solution of the dynamics of vortices whereas all our calculations are restricted to thermal equilibrium. Nevertheless, a simple estimate of the critical current along the lines of the Larkin Ovchinnikov argument can be made using only the equilibrium results. In the Larkin-Ovchinnikov theory, $R_{c}$ designates the distance beyond which the random force approximation breaks down. It is argued that for $r \gg R_{c}$ the vortices are pinned as independent bundles. In the Larkin-Ovchinnikov theory, the balance between random force due to disorder and the force $F_{c}$ due to the critical current implies that:

$$
F_{c}=\left(\frac{D}{R_{c}}\right)^{\frac{1}{2}}
$$

for vortices confined to planes parallel to the magnetic field in the presence of columnar disorder.

The distance beyond which vortices are uncorrelated is just the localization length of the bosons. Replacing $R_{c}$ with the boson localization length gives a power law dependence of the critical force on disorder. It can be checked first that in the limit $T \rightarrow 0$ one recovers the Larkin-Ovchinnikov result and second that the critical current goes to zero at the localization-delocalization transition for the bosons indicating the onset of dissipation in the vortex problem. In the pinned $2 \pi \rho_{0}$ vortex solid phase the critical current is given by:

$$
F_{c} \sim D^{\frac{8 T_{m}-T}{2\left(6 T_{m}-T\right)}}
$$

In the pinned $4 \pi \rho_{0}$ entangled vortex solid phase one has:

$$
F_{c} \sim \bar{D}^{\frac{2 T_{m}-T}{3 T_{m}-2 T}}
$$


The $4 \pi \rho_{0}$ Bose Glass does not extend down to $0 \mathrm{~K}$, so that there is no reason to expect recovering the Larkin-Ovchinnikov result for $T \rightarrow 0$. For $T<T_{m}$ it is replaced by the $2 \pi \rho_{0}$ Bose Glass as explained in section IVC3. At $T=T_{m}$, there is a transition from the $2 \pi \rho_{0}$ vortex liquid to the $4 \pi \rho_{0}$ vortex liquid. At that point(see figure 8), the critical current drops from $F_{c} \sim D^{7 / 10}$ for $T \rightarrow T_{m}^{-}$to $F_{c} \sim D$ for $T \rightarrow T_{m}^{+}$. This apparent discontinuity is a consequence of the approximations made. However, one expects a very rapid drop of $F_{c}$ as $T$ traverses $T_{m}$.

\section{Qualitative discussion of the strongly disordered case}

In the strongly disordered case, disorder can localize bosons before interchain hopping or interchain repulsion can open a gap in the antisymmetric degrees of freedom. In the case of vortices, this corresponds to independent pinning of vortices in each plane with neither interplane repulsion of vortices nor hopping of vortices. In such case, the two planes are completely decoupled and there is no correlation of vortex positions between the two planes. This phase can be seen as a pinned solid containing dislocations between the planes. This transition is the analogous of the transition between a Bragg glass phase and a vortex glass one occurring for point like disorder 3738. In the case of correlated disorder it corresponds to the transition between a dislocation free Bose glass (or Bragg Bose glas, 36 ) to the "standard" Bose glass containing dislocations. The condition for existence of that phase is that the pinning length of vortices in one plane is much shorter than both the correlation length due to interplane repulsion and the correlation length due to interplane hopping. This leads to:

$$
\begin{array}{r}
D \gg V^{\frac{3 T_{m}-T}{2 T_{m}-T}} \\
D \gg t_{\perp}^{\frac{T\left(3 T_{m}-T\right)}{2 T-T_{m}}}
\end{array}
$$

The resulting phase diagram is drawn on figure 9. The transition line between the phase with "superkinks" and the Bose Glass without dislocations corresponds to $V^{\frac{3 T_{m}-T}{2 T_{m}-T}}=t_{\perp} \frac{T\left(3 T_{m}-T\right)}{2 T-T_{m}}$. This vertical line ends at a point $D=t_{\perp} \frac{\frac{T\left(3 T_{m}-T\right)}{2 T-T_{m}}}{2 V^{\frac{3 T_{m}-T}{2 T_{m}-T}}}$. This suggests a possible multicritical point separating a Bose glass phase with dislocations, a Bose glass phase without dislocations (i.e. a Bragg Bose Glass) and a Bose Glass phase with "superkinks".

For $T>3 T_{m}$, the Bose glass phase with dislocation disappears and is replaced by a liquid phase. The transition line between the pinned entangled solid and the liquid phase starts at $T=\frac{3}{2} T_{m}$ for $D \rightarrow 0$. The melting temperature of the pinned entangled solid increases with disorder since the pinned solid gains energy from the random potential with respect to the liquid. The melting line of the Bose glass with dislocations can cut the melting line of the Bose glass with "superkinks". The intersection may correspond to a second multicritical point. The two possible multicritical points lie in the shaded zones of Fig. 9. It is possible to obtain the critical currents by the method of the previous section. One obtains:

$$
F_{c}=D^{\frac{4 T_{m}-T}{6 T_{m}-2 T}}
$$




\section{3. comparison of the one plane and the two plane vortex system}

For the two planes vortex system, pinning depends on two factors. The first one is of course temperature. At high enough temperatures, vortices always depin. The second factor is the relative magnitude of $t_{\perp}$ and $V . t_{\perp}$ depends on the potential that confines vortices in the planes but also on the temperature via the unperturbed wavefunction (see appendix for the case of a $\delta$ potential). $V$ depends both on temperature and vortex-vortex repulsion. It also depends on the potential that confines vortices in the plane via the unperturbed wavefunction. Therefore, the phase diagram of the non-disordered two planes system as a function of temperature is strongly affected by the potential that are chosen to model vortex-vortex interactions and vortex planes interactions. It is known from section IV B 1 that in the absence of disorder the two plane vortex system is in a vortex lattice state for $T<T_{m}$ and in a vortex liquid state for $T>T_{m}$. Also, from section IVB 1 we know that for $T<T_{m}$, we have a $2 \pi \rho_{0}$ Bose Glass, for $T_{m}<T<T_{L}$, a pinned $4 \pi \rho_{0}$ vortex lattice containing superkinks, and for $T>T_{L}$ vortices get unpinned.

Remarkably, the two temperature are related by the simple relation $T_{L}=\frac{3}{2} T_{m}$. It is also interesting to remark that at $T=T_{m}$ there is a discontinuity in vortex pinning length and in critical current. Of course, such a discontinuity is the result of the neglect of disorder with respect to the gap, and a more exact treatment would lead to a continuous albeit extremely rapid decrease of the critical current with temperature.

For the one plane system on the other hand, there is only one Bose glass phase and vortex depinning is solely driven by temperature. The transition Bose Glass to vortex liquid occurs at a higher temperature $T_{1}$ plane $=2 T_{L}$ and for given temperature and given amount of disorder the vortex pinning length is shorter in the one plane system if $T>T_{m}$. On the other hand, for $T<T_{m}$, the pinning length of the two planes system is the shortest due to the transition from the $4 \pi \rho_{0}$ to the $2 \pi \rho_{0}$ Bose glass as the temperature is lowered. As a consequence, for $T<T_{m}$ vortex pinning is the strongest in two planes systems whereas for $T>T_{m}$ it is the strongest in one plane systems. What distinguishes sharply one plane vortex systems from two planes vortex systems is the existence of a two step transition from the Bose glass phase to the vortex liquid. The first step is the formation of superkinks that halve the periodicity of the underlying lattice, leading to a second Bose glass phase much less well pinned by columnar disorder, and in a second step the melting of the new Bose glass phase.

All these considerations are valid for $T>0$. However, for $T=0$, thermal fluctuations are not available to screen the pinning potential. It is therefore interesting when comparing the one plane and the two plane system to consider the limit $T \rightarrow 0$. In the $T \rightarrow 0$ limit, it is possible to obtain the pinning length by an Imry-Ma 56 type argument. One requires that the phase of the field coupled to the random potential jumps by $2 \pi$ on a length $L$. This leads to a variation of $\phi_{s}$ on the length $L$ of $\delta \phi_{s} \pi \sqrt{2}$. The resulting pinning energy is:

$$
E(L)=\frac{2 \pi^{2} c_{1}}{L^{2}}-\sqrt{\frac{2 D}{L}}
$$

Minimizing with respect to $L$ gives: 


$$
L_{\text {pin. }, 2 \text { ch. }}=\left(\frac{32 \pi^{4} c_{1}^{2}}{D}\right)^{1 / 3}
$$

For one plane, the same procedure gives :

$$
L_{\text {pin., } 1 \text { ch. }}=\left(\frac{16 \pi^{4} c_{1}^{2}}{D}\right)^{1 / 3}
$$

The pinning length is therefore larger in the two plane system by a factor $2^{1 / 3}$ than in the one chain system.

Therefore, at $\mathrm{T}=0$, we expect two regimes: For $D^{1 / 3} \gg V^{1 / 2}$, the two planes are mutually decoupled and individually pinned on disorder. For $D^{1 / 3} \ll V^{1 / 2}$, the two planes are locked and the pinning length is larger than in a single plane system with the same disorder strength. This situation is reminiscent of the one that obtains in higher dimensions with a solid phase without dislocation at weak disorder and a more strongly pinned solid phase with dislocation at higher disorder. Such situation is expected on physical grounds, since forming dislocations allows the system to gain more energy from the random pinning potential.

At non-zero temperature, the solid phase with dislocations is more easily melt than the solid without dislocations. Therefore, one expects that the pinning length decreases faster with temperature in the phase in which the vortices are pinned independently in the planes than in the phase in which the vortices are pinned by the antisymmetric component of disorder. Such behavior is indeed what we have obtained for $T>0$.

\section{CONCLUSION}

In this paper, we have investigated two chains of strongly correlated bosons, coupled by boson interchain hopping and boson-boson repulsion, and applied our results both to quantum systems and to their classical analog of vortices in type II superconductors in the presence of columnar defects.

Using bosonization and renormalization techniques, we have obtained the phase diagram in the absence of disorder. It contains four different phases: two charge density wave phases of different periodicity, a conventional superfluid phase, and a superfluid phase made of pairs of bosons. This last superfluid phase is an analog of the an XY phase found in spin one 48 chains and spin ladders29. For weak inter chain interactions one finds a transition between a superfluid and "crystalline" (charge density wave) phase. This transition occurs for considerably more repulsive interactions $(K=1 / 2)$ than in the case of a single chain, where it would occur for hard core bosons $(K=1)$. Interchain hopping thus stabilizes considerably the superfluid phase. Paradoxically, the long wavelength part of the interchain repulsion favors hopping between the chains and thus also helps in stabilizing a superconducting phase.

We have then considered the effects of a random potential. For a single chain this leads to the localization of the bosons for moderately repulsive interactions $(K=3 / 2)$. In the case of the ladder the superfluid phase is much more resistant to localization. The transition occurs at more repulsive interactions $(K=3 / 4)$. In particular bosons with only contact interactions are now always superfluid. Two different localized phases exist: first a pinned 
charge density wave where interchain hopping occurs, then for more repulsive interactions ( $K<1 / 2)$, a pinned charge density wave where hopping is frozen and with "crystalline" order of the bosons. Interchain boson repulsion pushes the system towards localization. Using renormalization we have also extracted the localization length as a function of disorder strength and interactions, and the temperature and frequency dependence of the conductivity. For the ladder, the exponent of the superfluid correlation function takes at the transition the universal value $\eta=1 / 3$. The results for the compressibility and exponents obtained in our microscopic model are in agreement with the general scaling relations proposed for the superfluid-insulator transition 18 . Moreover, the superfluid correlation exponent $\eta$ for the ladder takes the same value than the one for a single chain16, suggesting the interesting possibility of a universal exponent of the superfluid-insulator transition in one dimension. Such a property would be much stronger than the simple inequality $\eta<1$ suggested by the scaling. For an even number of chains, we have given a general argument, valid when all the mode but one are gapped, that this is indeed the case. This conjecture remains of course to be tested in much more details, both for the case of an odd number of chains and for situations where some modes could remain gapless.

These results have a direct translation for the case of vortices in type II superconductors. The bosonic ladder corresponds here to two planes of vortices with both vortex-vortex interactions and hopping of vortices between the planes. In the boson vortex mapping superfluidity of the boson corresponds to a liquid state for the vortices, whereas the CDW phases correspond to crystalline order of the vortices. For the pure systems there is a transition between a high temperature liquid phase and a low temperature solid phase. The melting temperature $T_{m}$ is much smaller than the melting of a single plane due to the possibility of forming superkink between the planes. The transition is direct without an intermediate "supersolid" phase having both crystalline and superfluid properties, advocated to exist in this geometry ${ }^{4}$. This absence of supersolid phase could be due to our restriction to only two coupled planes instead of an infinite number. However since the existence of the supersolid was only advocated based on perturbative arguments that would also give such a phase for the ladder, our study of the ladder shows that the possible existence of such a phase needs to be put on a firmer basis. In addition to these two phases, stable for weak interplane interaction, two other phases can exist: a vortex solid but where the vortices form superkinks between planes and a bound vortex pairs liquid. The random potential in the bosonic system is equivalent to columnar defects for the vortices. In presence of disorder two different transitions take place. At low temperature one is in a pinned solid state with no hopping between the planes. This corresponds to a Bose glass state for the vortices without dislocations between planes (nicknamed Bragg Bose glass 36 ). Upon increasing the temperature a first transitions occurs at $T_{m}$ between the Bose glass and a phase where vortices hop between planes giving a (pinned) solid with half the periodicity of the low temperature one. Combining RG with Larkin-Ovchinnikov arguments allows to compute the critical currents. At this transition the critical current is strongly decreased but remains finite. This decrease of the critical current is typical of columnar disorder and would not occur for pinning by twins. The liquid, with zero critical current is only recovered at higher temperature $T=3 / 2 T_{m}$ both for twins and columnar defects. This increase of the melting temperature is reminiscent of what is expected for columnar defects in higher dimensionality. Note that in our one dimensional system even an infinitesimal disorder would cause a finite shift of the 
melting temperature.

If disorder is increased, the planes decouple or equivalently dislocations appear between them. One recovers in this case the properties of individually pinned planes. This leads to an increase of the critical current similar to the one occurring for point like disorder when going from a Bragg glass state to a vortex glass state 36 . The transition occurs here between a dislocation free Bose glass (Bragg Bose glass) and a conventional Bose glass. Upon increasing the temperature there is then a single melting to a liquid phase but at a much higher temperature $T^{\prime}=3 T_{m}$. One thus expects for correlated disorder the same topology of phase diagram as for point like disorder, with two different glass phases but with the difference that increasing the correlated disorder raises the melting temperature. Whether the superkink phase close to the liquid for weak disorder exists in a three dimensional system is an interesting question that would deserve more investigations.

The ladder geometry thus provide an interesting starting point to study competition between elasticity and disorder in vortex systems while retaining realistic feature. It would be interesting to investigate the effect of point disorder and the interplay of columnar and point disorder in that system. This has already been done for a single plane system 57 and it would be interesting to know how interplane hopping modifies the response of the system to competing disorder. Also, systems with more than two coupled planes could provide further insight in the properties of coupled vortex planes and may be relevant to experiments. Finally, a mean field theory investigation of coupled planes would help in comparing the results with experiments on vortex pinning in BiSSCO.

\section{ACKNOWLEDGMENTS}

We are grateful to D. Carpentier, P. Le Doussal and H.J. Schulz, . for many illuminating discussions.

\section{APPENDIX A: BOSONIZATION OF BOSONS}

In this section the semi-phenomenological bosonization procedure introduced in Ref. 47 to describe the low energy physics of one dimensional interacting bosons is reviewed briefly. The reader already familiar with this technique should read it quickly to get acquainted with the notations. In Ref. 47 it is argued that a one dimensional interacting boson system always has an associated bosonized Hamiltonian describing the low energy, long momentum phonon excitation of the system :

$$
H=\int \frac{d x}{2 \pi}\left[u K(\pi \Pi)^{2}+\frac{u}{K}\left(\partial_{x} \phi\right)^{2}\right]
$$

$\Pi$ and $\phi$ satisfy canonical commutation relations : $\left[\Pi(x), \phi\left(x^{\prime}\right)\right]=\imath \delta\left(x-x^{\prime}\right)$. According to ref. 47, the operators of the original bosonic theory can be expressed as a function of the fields entering the Hamiltonian (A1). The bosonized form of the boson annihilation operator in one dimension is : 


$$
\psi_{B}(x) \sim e^{\imath \theta(x)}\left(\sum_{m=-\infty}^{\infty} e^{2 \imath m\left(\phi(x)+\pi \rho_{0} x\right)}\right)
$$

where $\theta(x)=\pi \int_{-\infty}^{x} d x^{\prime} \Pi\left(x^{\prime}\right)$ and $a$ is a short distance cutoff.

The bosonized form for the density is :

$$
\rho_{B}(x)=\left(\rho_{0}+\frac{\partial_{x} \phi}{\pi}\right) \sum_{m=-\infty}^{\infty} e^{2 \imath m\left(\phi(x)+\pi \rho_{0} x\right)}
$$

where $\rho_{0}$ is the average boson density. Boson Green's functions and density density correlation functions are obtained by the standard methods of bosonization 1 , 58,59 giving:

$$
\begin{aligned}
\left\langle T_{\tau} e^{\imath n \theta(x, \tau)} e^{-\imath n \theta(0,0)}\right\rangle & =\left(\frac{1}{x^{2}+u^{2} \tau^{2}}\right)^{\frac{n^{2}}{4 K}} \\
\left\langle T_{\tau} e^{\imath 2 n \phi(x, \tau)} e^{-\imath 2 n \phi(0,0)}\right\rangle & =\left(\frac{1}{x^{2}+u^{2} \tau^{2}}\right)^{n^{2} K}
\end{aligned}
$$

The boson creation operator is also the order parameter for superfluidity and the $2 \pi \rho_{0}$ component of the density is the charge density wave order parameter. Thus, (A4) shows that in the one dimensional bosonic system there is no long range order (in agreement with the Mermin-Wagner theorem60.61), but only quasi long range order. In particular, the susceptibilities associated with these operators diverge in the long wavelength low frequency limit. When $K$ increases, the system has a stronger tendency to superfluidity, whereas when $K$ decreases it has a stronger tendency to charge density wave order.

$u, K$ still have to be determined as a function of the microscopic boson-boson interactions. To do so, one has to relate them to ground state parameters of the interacting system. In Galilean invariant systems, an exact relation between $u, K$ hold\$47 and simplifies considerably the situation. This relation is : $u K=\frac{\pi \rho_{0}}{m}$, where $m$ is the mass of a boson. Knowing this, only one relation between $u, K$ and a parameter of the ground state suffices to determine $u$ and $K$. A convenient quantity is the compressibility $\chi$ defined by:

$$
\chi=\frac{1}{L}\left(\frac{\partial^{2} E}{\partial^{2} L}\right)^{-1}
$$

E being the ground state energy of the system. The compressibility can also be expressed as a function of $u, K$ in the form:

$$
\chi=\frac{K}{\pi u \rho_{0}^{2}}
$$

and permits a determination of $u, K$ as a functions of the parameters of the microscopic Hamiltonian. $\chi$ contains all interaction effects for Galilean invariant systems. On physical ground, the higher the boson-boson repulsion, the lower the compressibility. Therefore, $K$ decreases when repulsion increases. According to (A4) this means that repulsive interactions tend to favor charge density wave order versus superfluidity. In the case of bosons interacting 
with a repulsive $\delta$-function potential, the ground state energy and the compressibility can be obtained exactly as a function of the interaction 62 . This allows for the determination of $u, K$. In the case of a more general interaction potential, one has to rely on numerical techniques such as Quantum Monte Carlo simulations 63 .

Bosons on a lattice lack Galilean invariance, so that $u K \neq \frac{\pi \rho_{0}}{m}$. Nevertheless, (A6) remains valid. To determine $u, K$ one needs another relation linking $u, K$ to a parameter of the ground state. One convenient parameter is the charge stiffness $\$ 64$ defined for a closed ring of perimeter $L$ by:

$$
\mathcal{D}=\frac{L}{2}\left(\frac{d^{2} E(\varphi)}{d^{2} \varphi^{2}}\right)_{\varphi=0}
$$

$\varphi$ being a flux threading the system. For bosons that do not carry electrical charge, it is nevertheless possible to define a "charge stiffness" by using the sensitivity of energy levels to boundary conditions since the only effect of the flux $\varphi$ amounts to a change of boundary conditions. From the definition (A7), one obtains :

$$
\mathcal{D}=u K
$$

In Galilean invariant systems, the charge stiffness is independent of the interactions. Combined with (A6) this equation allows to determine $u, K$ as a function of the original parameters of the Hamiltonian in the most general case. For instance, for hard core bosons on a lattice, using an exact mapping onto the XXZ spin $1 / 2$ chain in a magnetic field ${ }^{4}$ one can obtain an exact expression of $u, K$ using the Bethe Ansatz solution of the spin chain problem. The more general case of soft core boson needs to be treated numerically.

\section{APPENDIX B: SUPERFLUID-DISORDERED EXPONENT}

Let us consider a ladder with $p$ legs. The density on chain $i$ can be expressed in terms of the field $\phi_{i}$ as

$$
\rho_{i} \sim e^{2 \imath \phi_{i}}
$$

As for the two leg ladder it will be convenient to introduce the various modes (equivalent to the symmetric and antisymmetric ones) between the chains. If one now assumes that every mode except the totally symmetric one is gapped (the fact that the totally symmetric one remains always gapless is a consequence of the Galilean invariance), one can then proceed as for the two leg ladder. The totally symmetric mode has the following expression:

$$
\phi_{s}=\frac{1}{\sqrt{p}} \sum_{i=1}^{p} \phi_{i}
$$

And all the modes can be decomposed as:

$$
\phi_{i}=\frac{\phi_{s}}{\sqrt{p}}+\text { non-symmetric modes }
$$


Let us assume that all of the non-symmetric modes are gapped with an average value for the corresponding $\theta$ 's fields. The coupling to disorder should only retain the massless mode. It is thus necessary to form higher order terms in perturbation theory to cancel all the other modes. It is easy to check that the remaining coupling to disorder is of the form

$$
H=\int d x V(x) e^{\imath 2 p / \sqrt{p} \phi_{s} x}
$$

since it is necessary to go to $p$-th order to get rid of all the other modes. (B4) obviously reproduces the two leg result. Using the same $\mathrm{RG}$ argument the transition now occurs for $K=3 /(2 p)$, i.e. more and more repulsive interactions. Similarly the superfluid order parameter on chain $i$ is given by

$$
\psi_{i} \sim e^{\imath \theta_{i}}
$$

Using again the decomposition in the above modes and assuming that all gaped modes have a an average value for the $\theta$ field, one gets

$$
\psi_{i} \sim e^{\frac{2}{\sqrt{p}} \theta_{s}} C
$$

where $C$ is a universal constant coming from the massive modes. In that case the correlation function at the transition decays as

$$
\left\langle\psi_{i}(r) \psi_{i}(0)\right\rangle \sim\left(\frac{1}{r}\right)^{\frac{1}{2 p K}}
$$

giving the universal exponent $\eta=1 / 3$ regardless of the number of legs.

\section{APPENDIX C: DERIVATION OF THE HAMILTONIAN OF THE COUPLED PLANES IN THE TIGHT-BINDING APPROXIMATION}

The Hamiltonian for non-interacting bosons in a plane with two potential wells is :

$$
H=-\frac{\hbar^{2}}{2 m}\left(\nabla_{y}^{2}+\nabla_{x}^{2}\right)+V\left(y-Y_{1}\right)+V\left(y-Y_{2}\right)
$$

$V(x)$ represents an isolated potential well. Consider the Hamiltonian h:

$$
h=-\frac{\hbar^{2}}{2 m} \nabla_{y}^{2}+V(y)
$$

and call $\varphi(y)$ its eigenfunctions. In the tight binding approximation the eigenfunctions are sought in the approximate form :

$$
\Psi(x, y)=\psi(x)\left(\alpha \varphi\left(y-Y_{1}\right)+\beta \varphi\left(y-Y_{2}\right)\right)
$$

and the Hamiltonian is of the form $h+V$. The Hamiltonian is simplified into a Hamiltonian with a hopping term: $t_{\perp}=\int d y \varphi\left(y-Y_{1}\right) V\left(y-Y_{2}\right) \varphi\left(y-Y_{2}\right)$ In the case : $V(y)=-V_{0} \delta(y)$, it is possible to obtain the exact eigenfunctions of (C2) and calculate $t_{\perp}$ as : 


$$
t_{\perp}=-V_{0} \kappa \exp \left(-\kappa\left|Y_{1}-Y_{2}\right|\right)
$$

with $\kappa=\frac{m V_{0}}{\hbar^{2}}$. This allows a rewriting of the Hamiltonian in the second quantized representation as:

$$
H=\int d x\left[\Psi_{1}^{\dagger}(x)-\frac{\hbar^{2}}{2 m} \nabla_{x}^{2} \Psi_{1}(x)+\Psi_{2}^{\dagger}(x)-\frac{\hbar^{2}}{2 m} \nabla_{x}^{2} \Psi_{2}(x)+t_{\perp}\left(\Psi_{1}^{\dagger}(x) \Psi_{2}(x)+\text { H. с. }\right)\right]
$$

The wavefunction (second-quantized) being: $\Psi(x, y)=\Psi_{1}(x) \varphi\left(y-Y_{1}\right)+\Psi_{2}(x) \varphi\left(y-Y_{2}\right)$. Adding interactions leads to the following expression for intrachain interaction:

$$
u\left(x-x^{\prime}\right)=\int d y d y^{\prime} U\left(x-x^{\prime}, y-y^{\prime}\right)|\varphi(y)|^{2}\left|\varphi\left(y^{\prime}\right)\right|^{2}
$$

and for interchain interaction:

$$
v\left(x-x^{\prime}\right)=\int d y d y^{\prime} U\left(x-x^{\prime}, y-y^{\prime}\right)\left|\varphi\left(y-Y_{1}\right)\right|^{2}\left|\varphi\left(y^{\prime}-Y_{2}\right)\right|^{2}
$$

There are also interactions which do not conserve the number of bosons in one chain However, these interactions merely correct the hopping term. In the case of the delta-function potential well, and a delta function repulsion between vortices in the form:

$$
U\left(x-x^{\prime}, y-y^{\prime}\right)=U_{0} \delta\left(x-x^{\prime}\right) \delta\left(y-y^{\prime}\right)
$$

$u, v$ have the following expressions :

$$
\begin{array}{r}
u\left(x-x^{\prime}\right)=U_{0} \frac{\kappa}{8} \\
v\left(x-x^{\prime}\right)=U_{0} \frac{\kappa}{8}\left[e^{-4 \kappa\left|Y_{1}-Y_{2}\right|}+2 \kappa\left|Y_{1}-Y_{2}\right| e^{-2 \kappa\left|Y_{1}-Y_{2}\right|}\right]
\end{array}
$$

The tight binding approximation is well justified in the limit $\kappa\left|Z_{1}-Z_{2}\right| \gg 1$ only. Therefore in the case of delta functions interactions, interchain hopping dominates over interchain repulsion. This may change in the case of longer range interactions.

\section{APPENDIX D: DERIVATION OF THE COUPLING WITH DISORDER FOR THE TWO PLANE SYSTEM}

In the mapping of vortex planes onto coupled bosonic chains, introduction of columnar disorder in the two plane system is equivalent to introduction of a time independent random potential in the two chain system. The part of the Hamiltonian describing such disorder is:

$$
H_{\text {disorder }}=\int d x d y v(x, y) \Psi^{\dagger}(x, y) \Psi(x, y)
$$

with:

$$
\overline{v(x, y) v\left(x^{\prime}, y^{\prime}\right)}=D \delta\left(x-x^{\prime}\right) \delta\left(y-y^{\prime}\right)
$$


y being the coordinate perpendicular to the chains and $\Psi$ being the full second quantized wave function. The chains being treated in the Tight Binding Approximation, one has:

$$
\Psi(x, y)=\psi_{1}(x) \varphi\left(y-Y_{1}\right)+\psi_{2}(x) \varphi\left(y-Y_{2}\right)
$$

Porting (D3) into (D1) leads to the following coupling:

$$
H_{\text {disorder }}=\int d x\left(v_{1}(x) \psi_{1}^{\dagger} \psi_{1}(x)+v_{2}(x) \psi_{2}^{\dagger} \psi_{2}(x)\right)+\int d x \delta t_{\perp}(x)\left(\psi_{1}^{\dagger} \psi_{2}(x)+\psi_{2}^{\dagger} \psi_{1}(x)\right)
$$

With:

$$
\begin{array}{r}
v_{2}(x)=\int d y v(x, y) \varphi^{2}\left(y-Y_{2}\right) \\
v_{1}(x)=\int d y v(x, y) \varphi^{2}\left(y-Y_{1}\right) \\
t_{\perp}(x)=\int d y v(x, y) \varphi^{2}\left(y-Y_{2}\right) \phi^{2}\left(y-Y_{1}\right)
\end{array}
$$

These definitions lead to:

$$
\begin{array}{r}
\overline{v_{1}(x) v_{1}\left(x^{\prime}\right)}=\overline{v_{2}(x) v_{2}\left(x^{\prime}\right)}=D \delta\left(x-x^{\prime}\right) \int d y \varphi^{4}(y) \\
\overline{v_{1}(x) v_{2}\left(x^{\prime}\right)}=D \delta\left(x-x^{\prime}\right) \int d y \varphi^{2}\left(y-Y_{1}\right) \varphi^{2}\left(y-Y_{2}\right) \\
\overline{\delta t_{\perp}(x) \delta t_{\perp}\left(x^{\prime}\right)}=D \delta\left(x-x^{\prime}\right) \varphi_{1}^{2}(y) \varphi_{2}^{2}(y)
\end{array}
$$

If the potential that confines vortices in the plane is of the form $-V_{0}\left(\delta\left(y-Y_{1}\right)+\delta\left(y-Y_{2}\right)\right)$, one can obtain an explicit form of the integrals in (D6). Introducing $\kappa=\frac{m V_{0}}{\hbar^{2}}$, one has $\varphi(y)=\sqrt{\kappa} e^{-\kappa y}$ and :

$$
\begin{array}{r}
\int_{-\infty}^{\infty} d y \varphi^{4}(y)=\frac{\kappa}{2} \\
\int_{-\infty}^{\infty} d y \varphi^{2}\left(y-Y_{1}\right) \varphi^{2}\left(y-Y_{2}\right)=\kappa\left(1+\frac{\kappa a}{2}\right) e^{-2 \kappa a}
\end{array}
$$

with $a=\left|Z_{1}-Z_{2}\right|$. The tight binding approximation being valid only for $\kappa a \gg 1, v_{1}$ and $v_{2}$ are almost uncorrelated. Also, disorder induces a very weak random interchain hopping. For the tight binding approximation to be valid, the random interchain hopping has to be much smaller than $t_{\perp}$. More precisely, the correlation length induced by the random hopping terms has to be much larger than the correlation length induced by $t_{\perp}$. We know that : $t_{\perp}=-\kappa V_{0} \exp \left(-\kappa\left|Z_{1}-Z_{2}\right|\right)$. This leads to the following criterion:

$$
\left(\frac{1}{t_{\perp}}\right)^{\frac{2}{4-K_{a}}} \ll\left(\frac{1}{D}\right)^{\frac{1}{3-K_{a}}}
$$

In terms of $V_{0}$ the criterion reads :

$$
D \ll \frac{\left(\kappa V_{0}\right)^{\frac{2\left(3-K_{a}\right)}{4-K_{a}}}}{\kappa\left(1+\frac{\kappa a}{2}\right) e^{\frac{2 \kappa a}{\left(4-K_{a}\right)}}}
$$

Since $\kappa a \gg 1$ when the tight binding approximation is correct, the condition on $D$ is always satisfied as long as the tight binding approximation is correct. 


\section{APPENDIX E: RELATION BETWEEN ELASTIC CONSTANTS OF A ONE VORTEX PLANE SYSTEM AND THE PARAMETERS OF ITS EQUIVALENT BOSONIC THEORY}

We consider a plane containing vortices. Let $\rho_{0}$ the vortex density, and $c_{44}$ and $c_{1}$ the elastic constants of the vortex system. The elastic Hamiltonian of the vortex system is:

$$
H=\frac{1}{2} \int d x d z\left[c_{44}\left(\partial_{z} u\right)^{2}+c_{1}\left(\partial_{x} u\right)^{2}\right]
$$

The Euclidean action of the equivalent bosonic system is:

$$
S=\int \frac{d x d \tau}{2 \pi K}\left[\frac{\left(\partial_{\tau} \phi\right)^{2}}{u}+u\left(\partial_{x} \phi\right)^{2}\right]
$$

Identifying the path integrals, and reminding that $\phi(x)=\pi \rho_{0} u(x)$ one finds:

$$
\begin{gathered}
c_{44}=T \frac{\pi \rho_{0}^{2}}{u K} \\
c_{1}=T \frac{\pi u \rho_{0}^{2}}{K}
\end{gathered}
$$

$c_{1}$ is the inverse compressibility both of the vortex system and the boson system. Also, for a translationally invariant vortex system: $c_{44}=\rho_{0} m$ Finally, $K=\frac{\pi \rho_{0}^{2} T}{\sqrt{c_{44} c_{1}}}$

\section{APPENDIX F: ABSENCE OF A SUPERSOLID PHASE IN THE TWO PLANE VORTEX SYSTEM}

The discussion is done one the equivalent boson Hamiltonian. At the point $K_{a}=1 / 2$, a refermionization of $H_{a}$ can be performed 6 . One obtains:

$$
H_{a}=\int \frac{d x}{2 \pi} v_{F}\left[(\pi \Pi)^{2}+\left(\partial_{x} \phi\right)^{2}\right]+\frac{t_{\perp}}{\pi \alpha} \int \cos \left(2 \tilde{\theta_{a}}\right)+\frac{g}{(\pi \alpha)^{2}} \int \cos \left(2 \tilde{\phi}_{a}\right)
$$

Where: $\tilde{\phi}_{a}=\sqrt{2} \phi_{a}$ and $\theta_{a}=\sqrt{2} \tilde{\theta}_{a}$.

Introducing the fermion fields $\Psi_{R, L}=\frac{e^{\imath\left(\tilde{\theta_{a}}+\tilde{\phi_{a}}\right)}}{\sqrt{2 \pi \alpha}}$, one obtains the fermionized Hamiltonian 65.

$H=-\imath v_{F} \int d x\left(\Psi_{R}^{\dagger} \partial_{x} \Psi_{R}-\Psi_{L}^{\dagger} \partial_{x} \Psi_{L}\right)+2 t_{\perp} \int d x\left(\Psi_{R}^{\dagger} \Psi_{L}^{\dagger}+\Psi_{L} \Psi_{R}\right)+\frac{g}{\pi \alpha} \int d x\left(\Psi_{R}^{\dagger} \Psi_{L}+\Psi_{L}^{\dagger} \Psi_{R}\right)$

Such Hamiltonian can easily be diagonalized 5 . It is clear that expectation values such as: $\left\langle\Psi_{R}\right\rangle$ are zero on the ground state of this Hamiltonian due to the Mermin-Wagner theorem - This implies $\left\langle\sin \left(\sqrt{2} \phi_{a}\right) \cos \left(\sqrt{2} \theta_{a}\right)\right\rangle=0$. If one could have $\left\langle\theta_{a}^{2}\right\rangle,\left\langle\phi_{a}^{2}\right\rangle,<\infty$ one would expect such quantity to be non-zero. This indicates that the presence of a supersolid phase in the two chain system is impossible. In a system of many coupled chains, however, the Mermin-Wagner does not apply and we cannot rule out a possible supersolid phase. 


\section{TABLES}

TABLE I. the 4 sectors of the two coupled bosonic chains model

\begin{tabular}{ccccc}
\hline \hline & I & II & III & IV \\
\hline$K_{s}$ & $<1$ & $<1 / 4$ & $>1$ & $>1 / 4$ \\
$K_{a}$ & $<1 / 2$ & $>1 / 2$ & $<1 / 2$ & $>1 / 2$ \\
\hline$\theta_{a}, \phi_{a}$ & $\left\langle\phi_{a}\right\rangle=\frac{\pi}{\sqrt{8}}$ & $\left\langle\theta_{a}\right\rangle=0$ & $\left\langle\phi_{a}\right\rangle=\frac{\pi}{\sqrt{8}}$ & $\left\langle\theta_{a}\right\rangle=0$ \\
phase & $\mathrm{CDW}-$ & $C D W^{4 \pi \rho_{0}}$ & $\mathrm{BPSF}$ & $\mathrm{SF}+$ \\
Order Parameter & $e^{\imath \sqrt{2} \phi_{s}}$ & $e^{\imath \sqrt{8} \phi_{s}}$ & $e^{\imath \sqrt{2} \theta_{s}}$ & $e^{\imath \frac{\theta_{s}}{\sqrt{2}}}$ \\
\hline \hline
\end{tabular}




\section{FIGURES}

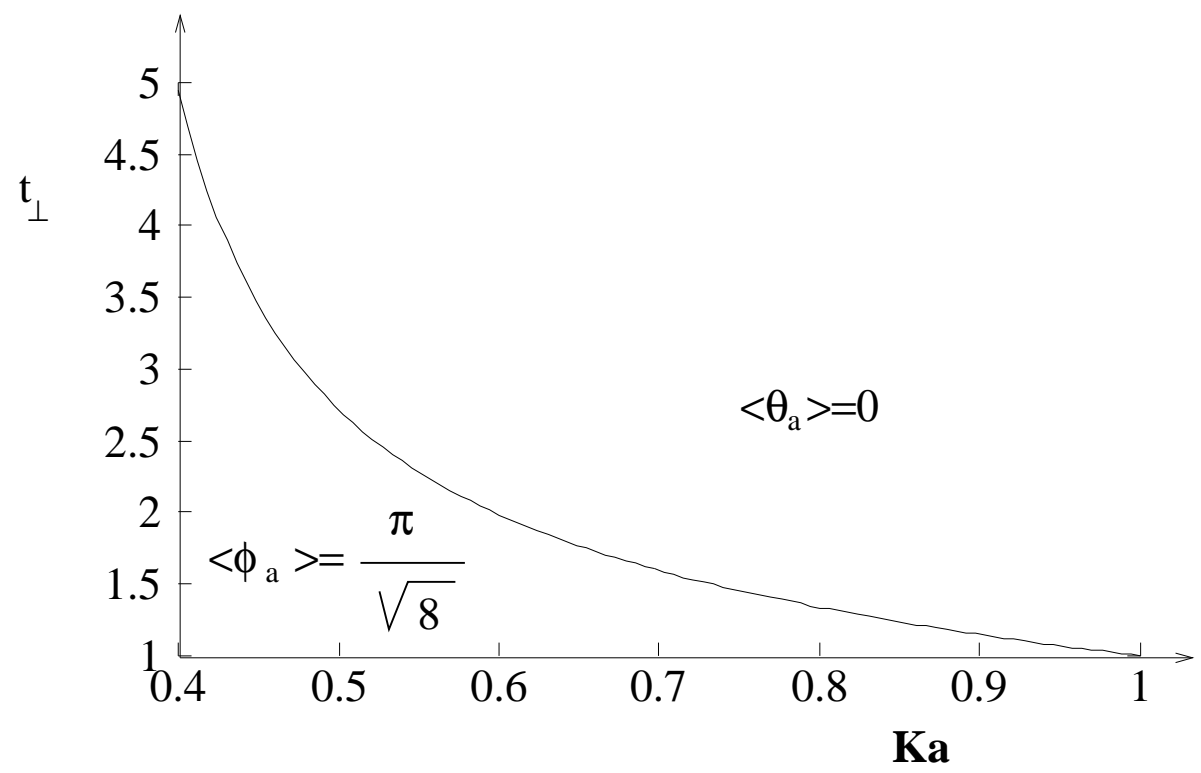

FIG. 1. The schematic phase diagram in the antisymmetric degrees of freedom as a function of $K_{a}$ and $t_{\perp}$. The line separates the phase with $\left\langle\phi_{a}^{2}\right\rangle<\infty$ from the phase with $\left\langle\theta_{a}^{2}\right\rangle<\infty$. For $K_{a}>1$ the system cannot sustain a phase with $\left\langle\phi_{a}^{2}\right\rangle<\infty$. For $1 / 4<K_{a}<1$ a nonzero $t_{\perp}$ is needed in order to stabilize a phase with $\left\langle\theta_{a}^{2}\right\rangle<\infty$. For $K_{a}<1 / 4$, the only possible phase is the phase with $\left\langle\phi_{a}\right\rangle=\frac{\pi}{\sqrt{8}}$

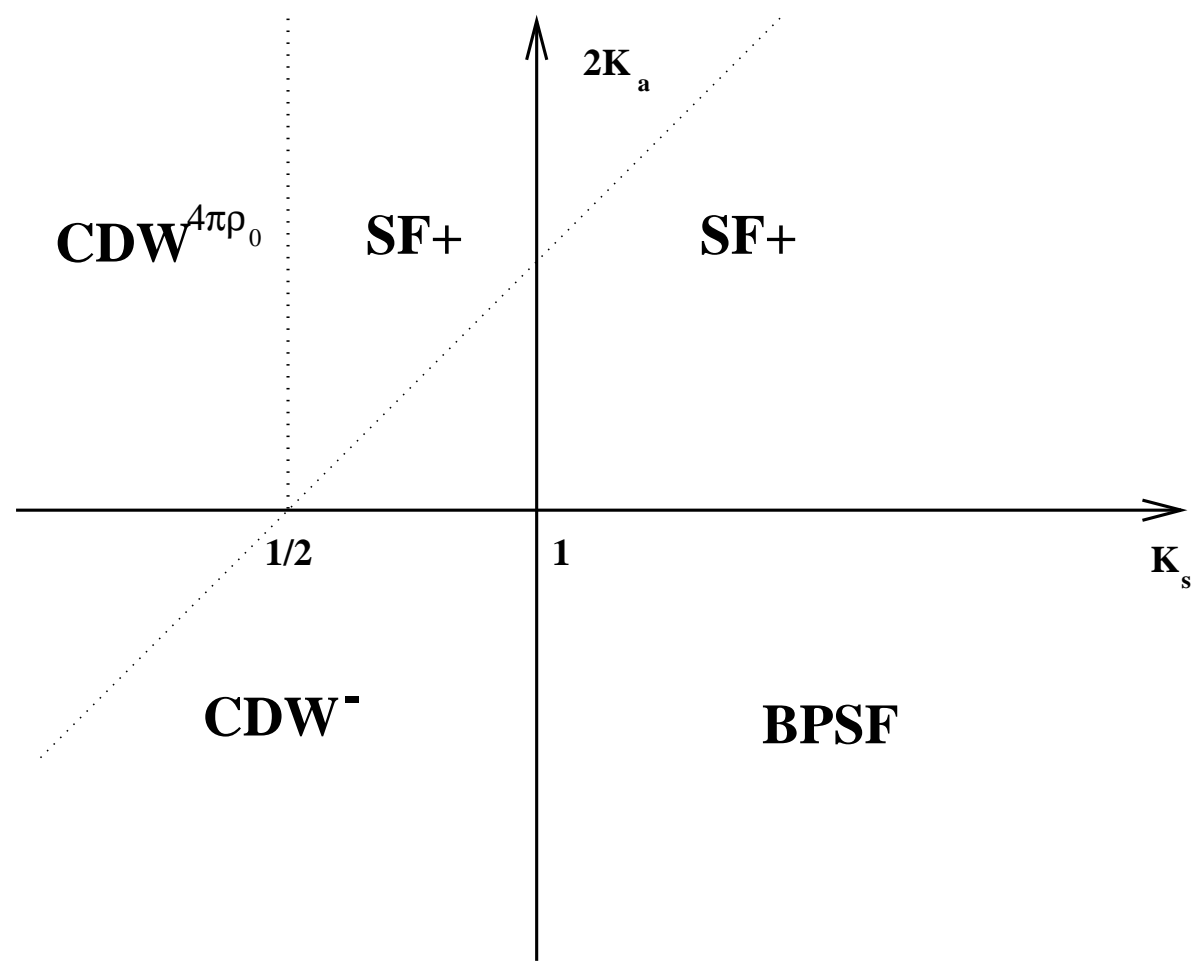


FIG. 2. The phase diagram of the two chain bosonic system without disorder. The dashed line separates the domain of dominant $\mathrm{CDW}^{4 \pi \rho_{0}}$ from the domain of dominant $\mathrm{SF}+$ fluctuations. The separation between dominant CDW- fluctuations and dominant BPSF fluctuations is at $K_{s}=1$. the dotted line corresponds to weakly coupled bosonic chains.

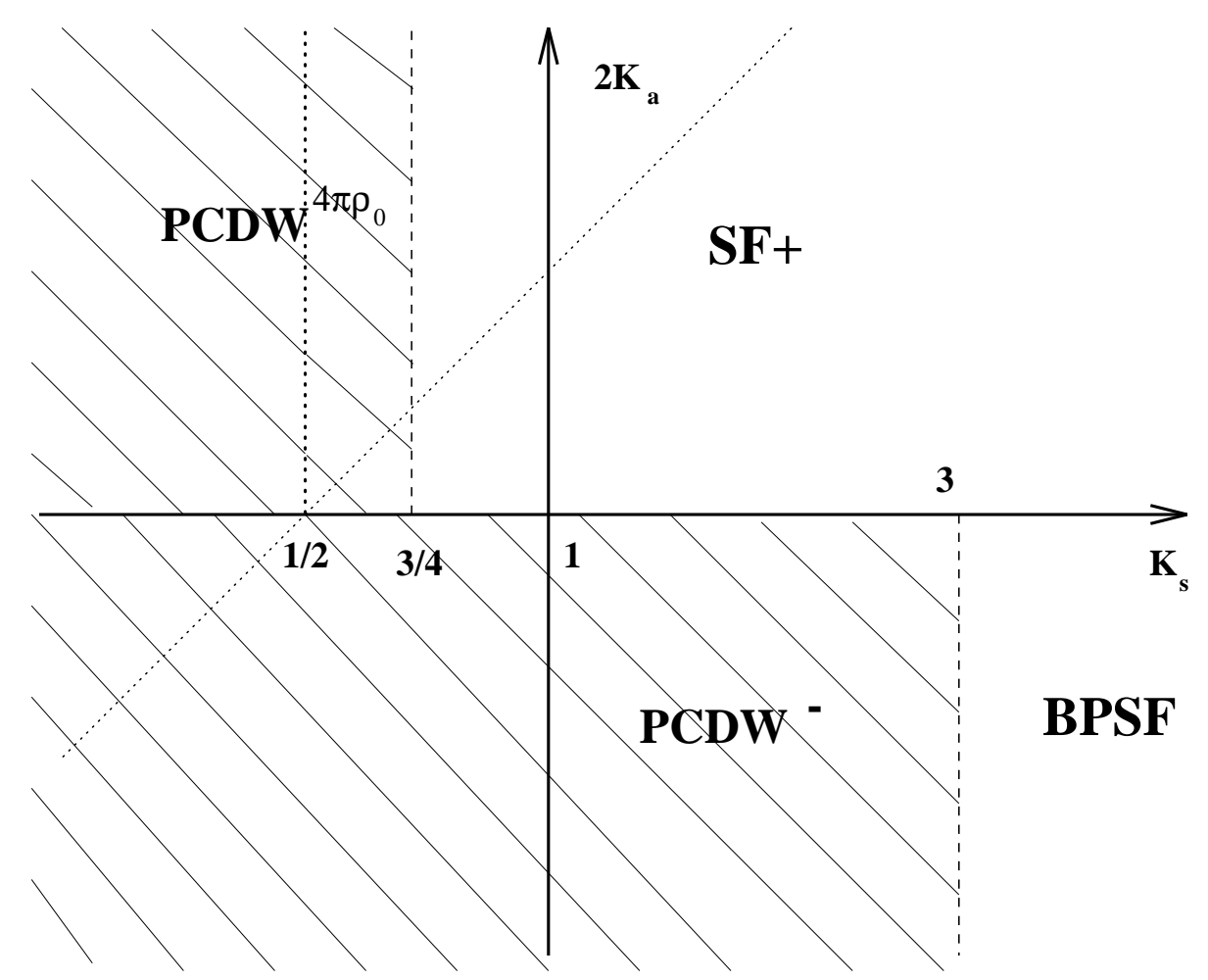

FIG. 3. The phase diagram of the two chain bosonic system in the presence of a random potential. The dotted line corresponds to weakly coupled bosonic chains. The hashed zones correspond to disordered phases in which bosons are localized. These disordered phases are pinned charge density waves. The stabilization of charge density waves by disorder permits to have a disordered $4 \pi \rho_{0}$ charge density wave for the two weakly coupled chains. 

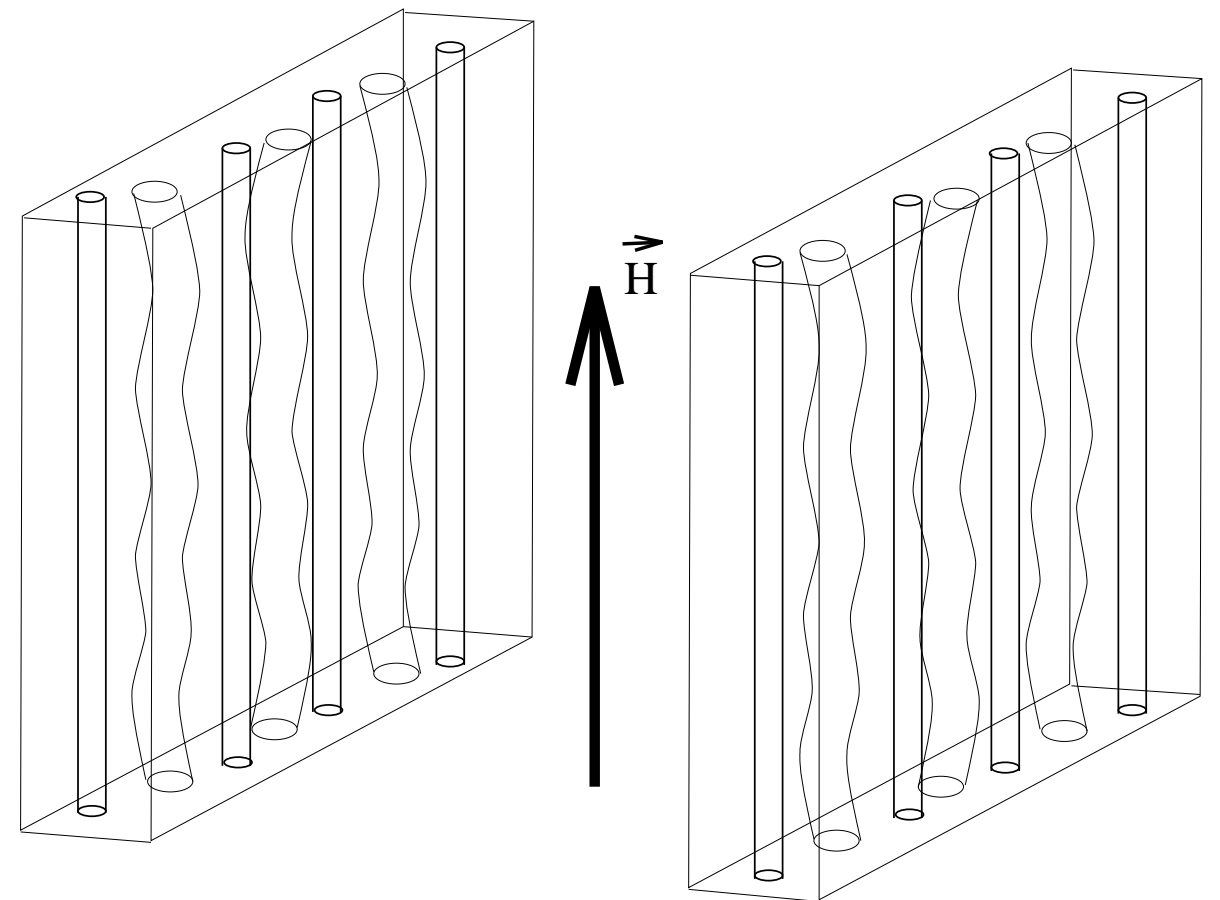

FIG. 4. The two planes vortex system. The vortices are confined in the planes and can pin on columnar defects parallel to the magnetic field. In the situation represented on the figure, the temperature is low and the vortices are confined to the planes 


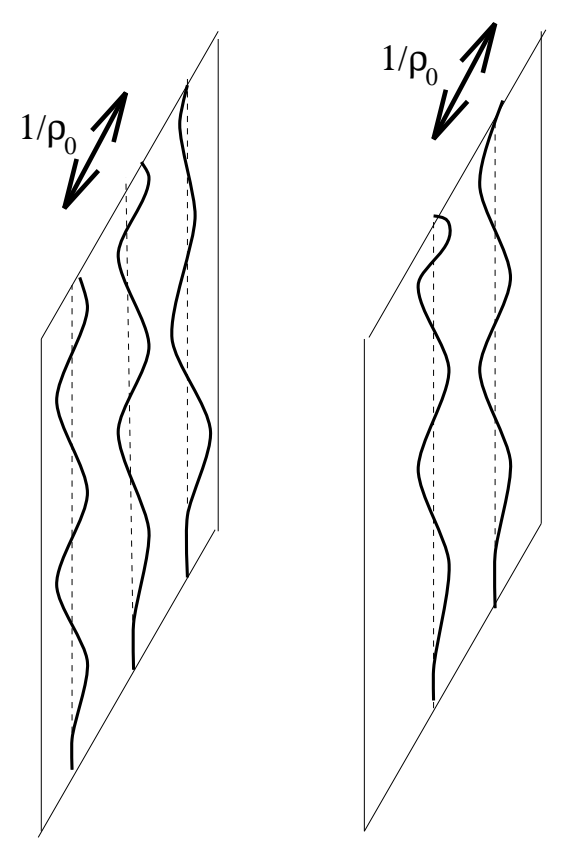

(a)

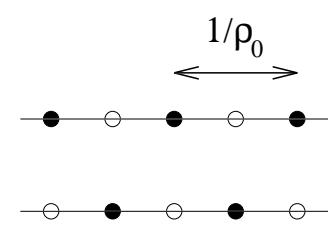

(b)

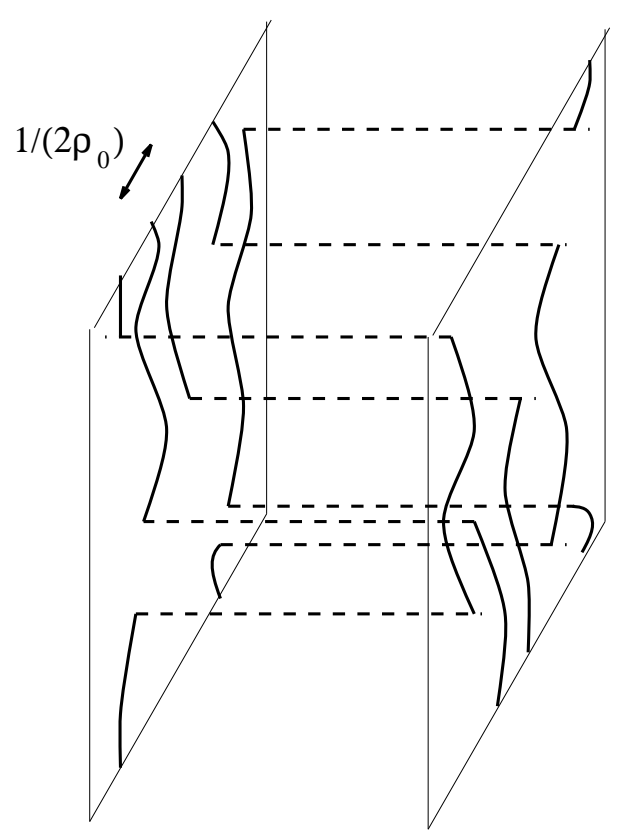

(c)

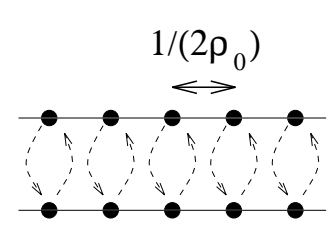

(d)

FIG. 5. The behavior of vortices in the $2 \pi \rho_{0}$ solid and in the $4 \pi \rho_{0}$ solid. (a) In the $2 \pi \rho_{0}$ solid, the vortices are confined in the planes and the average distance between two vortices is $1 / \rho_{0}$.

(b) Top view of the $2 \pi \rho_{0}$ solid.

(c) In the $4 \pi \rho_{0}$ solid, vortices are hopping from one plane to the other, leading to a halving of the distance between two maximums of the vortex density.

(d) Top view of the $4 \pi \rho_{0}$ solid. 


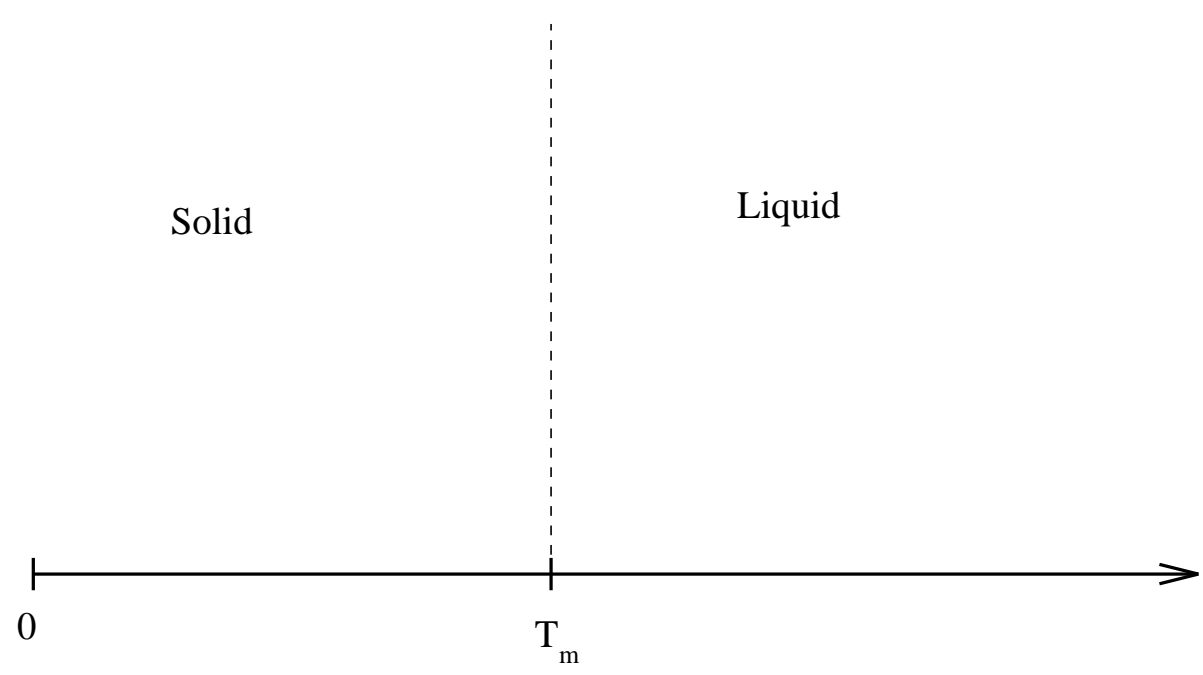

FIG. 6. The phase diagram of the pure two coupled planes vortex system. For $T<T_{m}$ one has the solid phase, with vortices confined in the planes, and for $T>T_{m}$, one has the liquid phase with vortices hopping from plane to plane.

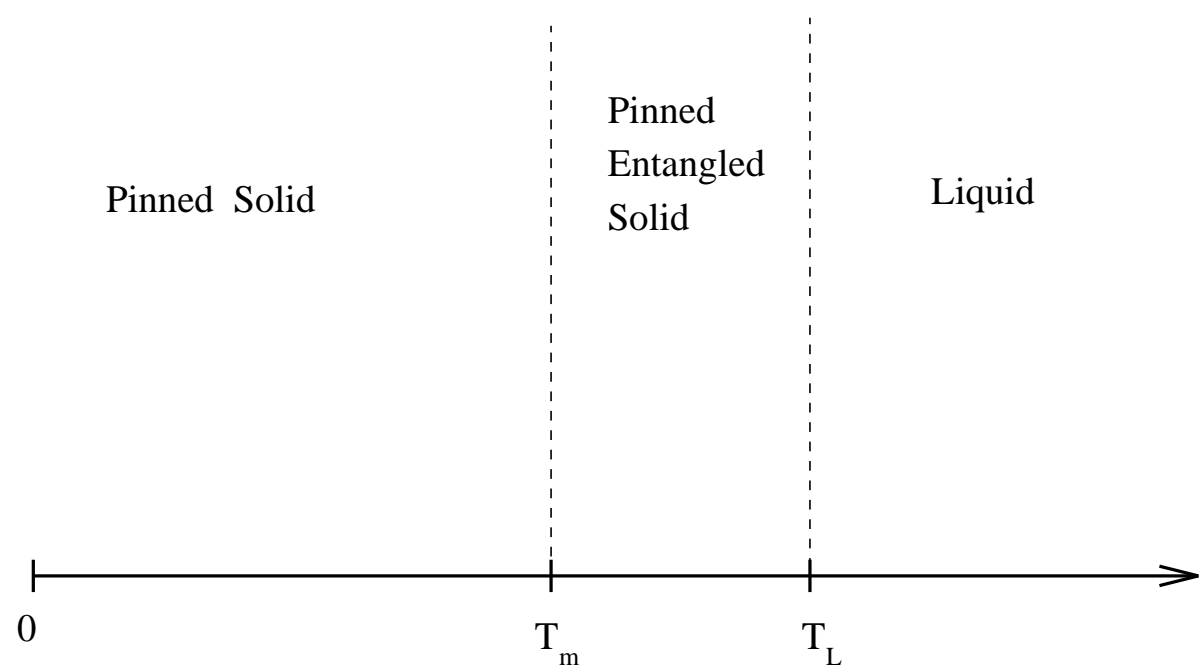

FIG. 7. The phase diagram of the weakly disordered two coupled planes vortex system. For $T<T_{m}$, one has the pinned solid phase, for $T_{m}<T<T_{L}=\frac{3}{2} T_{m}$, one has the entangled pinned solid, in which vortex pinning is weaker and vortices can hop from plane to plane. For $T>T_{L}$, one goes into a vortex liquid phase in which vortices are completely unpinned. 


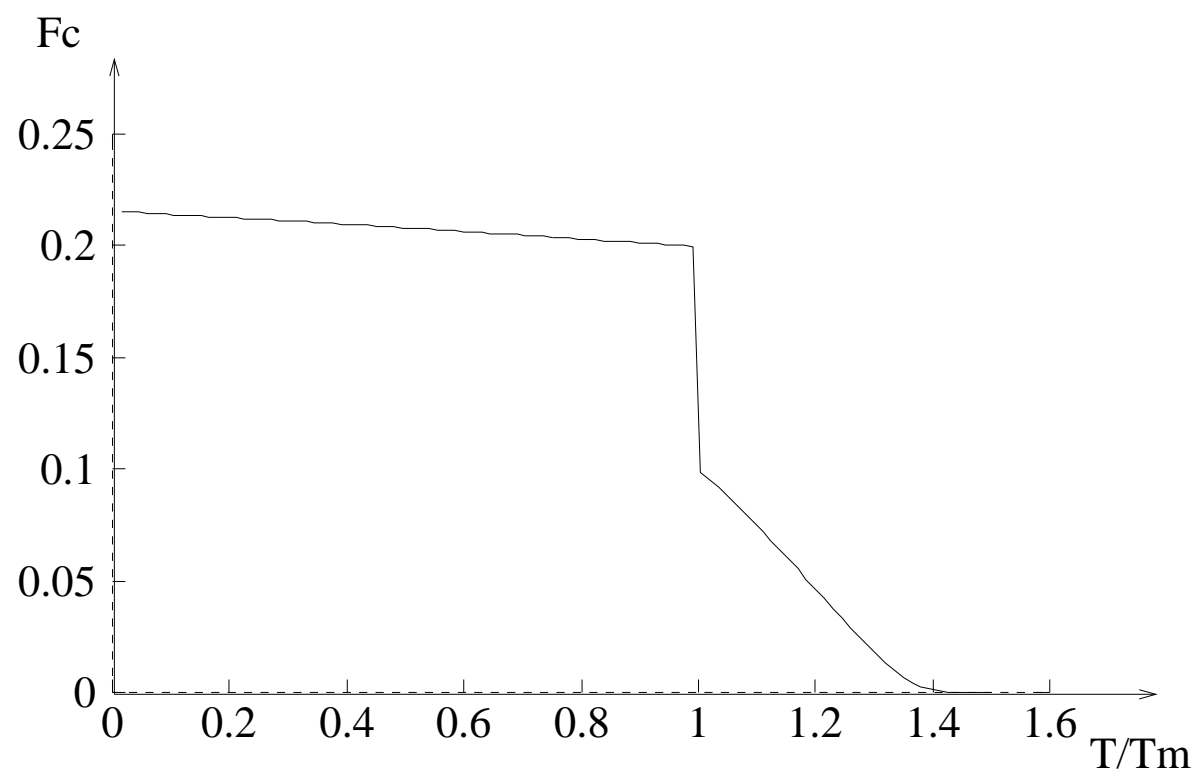

FIG. 8. Dependence of critical current upon temperature for $D=0.1$. For $T>T_{m}$, entanglement appears and the critical current is strongly reduced. For $T>\frac{3}{2} T_{m}$, the entangled solid is melt leading to a zero critical current.

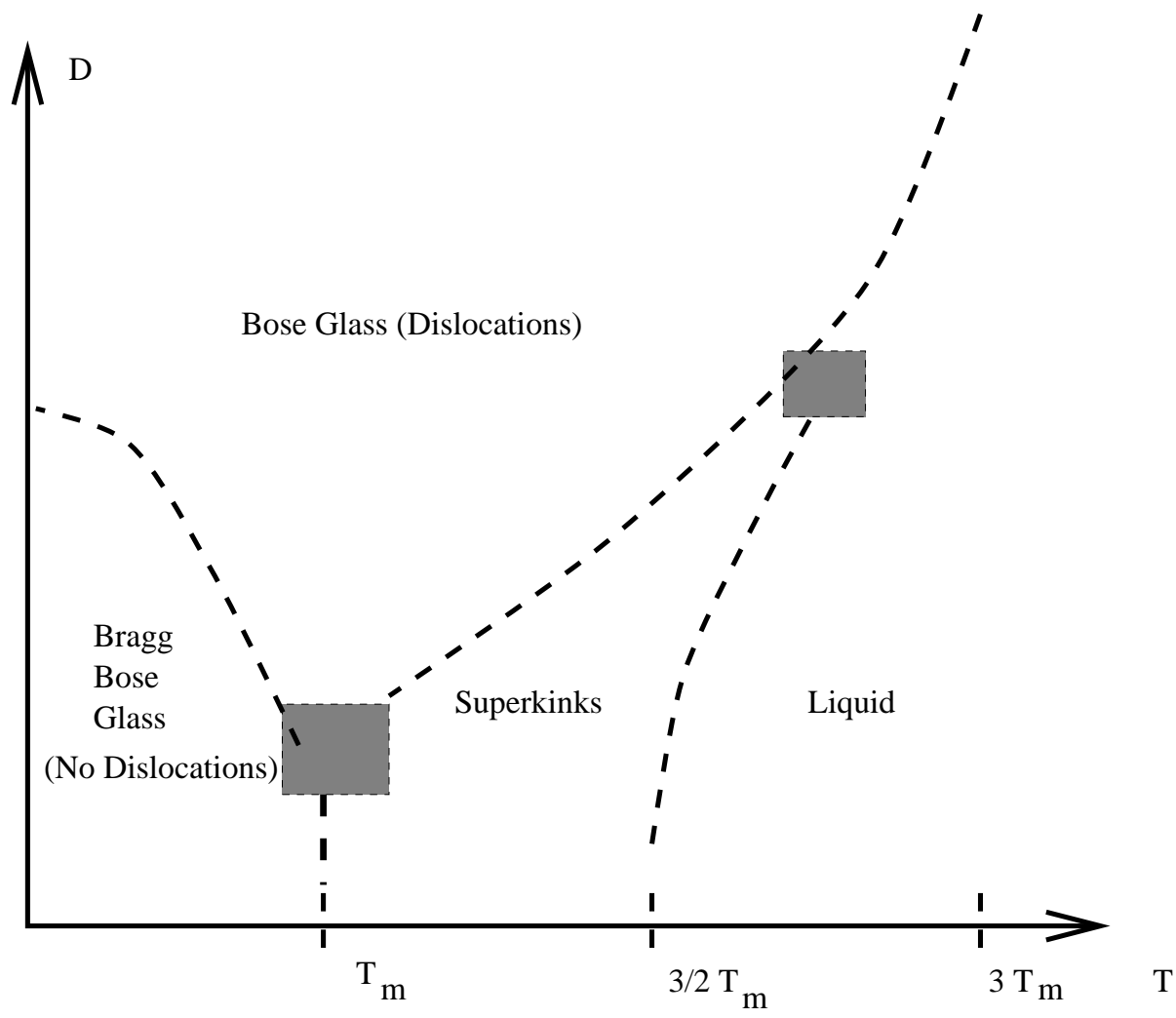


FIG. 9. Phase diagram as a function of temperature and disorder. For large disorder, a Bose glass with dislocations is formed. At weak disorder, depending on temperature, there is a Bragg Bose glass (without dislocations), an entangled Bose Glass and a vortex liquid. The shaded squares correspond to regions where a multicritical point may lie. 


\section{REFERENCES}

${ }^{1}$ Unité Mixte de Recherche du C. N. R. S.

${ }^{2}$ P. A. Lee and T. V. Ramakhrishnan, Rev. Mod. Phys. 57, 287 (1985).

${ }^{3}$ M. H. W. Chan et al., Phys. Rev. Lett. 61, 1950 (1988).

${ }^{4}$ A. Gerber and G. Deutscher, Phys. Rev. Lett. 63, 1184 (1989).

${ }^{5}$ S. J. Lee and J. B. Ketterson, Phys. Rev. Lett. 64, 3078 (1990).

${ }^{6}$ A. F. Hebard and M. A. Paalanen, Phys. Rev. Lett. 65, 927 (1990).

${ }^{7}$ M. A. Paalanen, A. F. Hebard, and R. R. Ruel, Phys. Rev. Lett. 69, 1604 (1992).

${ }^{8}$ A. van Oudenaarden and J. E. Mooij, Phys. Rev. Lett. 76, 4947 (1996).

${ }^{9}$ A. van Oudenaarden, S. . J. K. Várdy, and J. Mooij, Phys. Rev. Lett. 77, 4257 (1996).

${ }^{10}$ G. Blatter et al., Rev. Mod. Phys. 66, 1125 (1994).

${ }^{11}$ J. V. W. P. L. Gammel, L. F. Schneemeyer and D. J. Bishop, Phys. Rev. Lett. 61, 1666 (1988).

12 M. Charalambous, J. Chaussy, and P. Lejay, Phys. Rev. B 45, 5091 (1992).

${ }^{13}$ P. Le Doussal and D. R. Nelson, Physica C 232, 69 (1994).

${ }^{14}$ D. R. Nelson and P. Le Doussal, Phys. Rev. B 42, 10113 (1990).

${ }^{15}$ D. R. Nelson and V. M. Vinokur, Phys. Rev. B 48, 13060 (1993).

${ }^{16}$ T. Giamarchi and H. J. Schulz, Phys. Rev. B 37, 325 (1988).

${ }^{17}$ R. T. Scalettar, G. G. Batrouni, and G. T. Zimanyi, Phys. Rev. Lett. 66, 3144 (1991).

${ }^{18}$ M. P. A. Fisher, P. B. Weichman, G. Grinstein, and D. S. Fisher, Phys. Rev. B 40, 546 (1989).

${ }^{19}$ W. Krauth, N. Trivedi, and D. Ceperley, Phys. Rev. Lett. 67, 2307 (1991).

${ }^{20}$ E. S. Sorensen, M. Wallin, S. M. Girvin, and A. P. Young, Phys. Rev. Lett. 69, 828 (1992).

${ }^{21}$ M. Makivic, N. Trivedi, and S. Ullah, Phys. Rev. Lett. 71, 2307 (1993).

${ }^{22}$ M. Wallin and S. M. Girvin, Phys. Rev. B 47, 14642 (1993).

${ }^{23}$ F. Pázmándi, G. T. Zimanyi, and R. T. Scalettar, Phys. Rev. Lett. 75, 1356 (1995).

${ }^{24}$ L. Balents and M. P. A. Fisher, Phys. Rev. B 53, 12133 (1996).

${ }^{25}$ H. J. Schulz, Phys. Rev. B 53, 2959 (1996).

${ }^{26}$ S. P. Strong and A. J. Millis, Phys. Rev. Lett. 69, 2419 (1992).

${ }^{27}$ E. Orignac and T. Giamarchi, Phys. Rev. B 53, 10453 (1996).

${ }^{28}$ E. Orignac and T. Giamarchi, Phys. Rev. B 56, 7167 (1997).

${ }^{29}$ E. Orignac and T. Giamarchi, accepted for publication in Phys. Rev. B (unpublished).

${ }^{30}$ W. Jiang et al., Phys. Rev. Lett. 72, 550 (1994).

${ }^{31}$ R. C. Budhani, W. L. Holstein, and M. Sunenaga, Phys. Rev. Lett. 72, 566 (1994).

${ }^{32}$ A. V. Samoilov, M. V. Feigel'man, M. Konczykovski, and F. Holtzberg, to be published in Phys. Rev. Lett.

33 T. Giamarchi and P. Le Doussal, Phys. Rev. B 53, 15206 (1996).

34 T. Giamarchi and P. Le Doussal, Phys. Rev. Lett. 72, 1530 (1994).

35 T. Giamarchi and P. Le Doussal, Phys. Rev. B 52, 1242 (1995).

36 T. Giamarchi and P. Le Doussal, Phys. Rev. B 55, 6577 (1997).

${ }^{37}$ D. Carpentier, P. Le Doussal, and T. Giamarchi, Europhys. Lett. 35, 379 (1996).

38 J. Kierfeld, T. Nattermann, and T. Hwa, Phys. Rev. B 55, 626 (1997).

${ }^{39}$ D. S. Fisher, Phys. Rev. Lett. 78, 1964 (1997).

${ }^{40}$ M. J. P. Gingras and D. A. Huse, Phys. Rev. B 53, 15193 (1996). 
${ }^{41}$ A. Golub and B. Horovitz, Europhys. Lett. 39, 79 (1997).

${ }^{42}$ G. Jakob et al., Europhys. Lett. 19, 135 (1992).

${ }^{43}$ Z. Z. Li, H. Rifi, S. Megtert, and H. Raffy, Phys. Rev. Lett. 72, 4033 (1994).

${ }^{44}$ J. M. Triscone et al., Phys. Rev. Lett. 64, 804 (1990).

${ }^{45}$ L. Balents and D. R. Nelson, Phys. Rev. B 52, 12951 (1995).

${ }^{46}$ T. Giamarchi, Phys. Rev. B 46, 342 (1992).

${ }^{47}$ F. D. M. Haldane, Phys. Rev. Lett. 47, 1840 (1981).

${ }^{48}$ H. J. Schulz, Phys. Rev. B 34, 6372 (1986).

${ }^{49}$ M. E. fisher, Rep. Prog. Phys. 30, 615 (1967), and references therein.

${ }^{50}$ C. Doty and D. S. Fisher, Phys. Rev. B 45, 2167 (1992).

${ }^{51}$ T. Giamarchi, Phys. Rev. B 44, 2905 (1991).

${ }^{52}$ C. Kane and M. P. A. Fisher, Phys. Rev. Lett. 68, 1220 (1992).

${ }^{53}$ C. Kane and M. P. A. Fisher, Phys. Rev. B 46, 15233 (1992).

${ }^{54} \mathrm{~T}$. Giamarchi and H. Maurey, in Correlated fermions and transport in mesoscopic systems, edited by T. Martin, G. Montambaux, and J. Tran Thanh Van (Editions Frontières, Gif sur Yvette, France, 1996).

${ }^{55}$ A. I. Larkin and V. M. Vinokur, Phys. Rev. Lett. 75, 4666 (1995).

${ }^{56}$ H. Fukuyama and P. A. Lee, Phys. Rev. B 17, 535 (1978).

${ }^{57}$ T. Hwa, D. R. Nelson, and V. M. Vinokur, Phys. Rev. B 48, 1167 (1993).

${ }^{58}$ J. Sólyom, Adv. Phys. 28, 209 (1979).

${ }^{59}$ V. J. Emery, in Highly Conducting One-Dimensional Solids, edited by J. T. Devreese and al. (Plenum, New York, 1979), p. 327.

${ }^{60}$ N. D. Mermin and H. Wagner, Phys. Rev. Lett. 17, 1133 (1967).

${ }^{61}$ N. D. Mermin, Phys. Rev. 176, 250 (1968).

${ }^{62}$ E. H. Lieb and W. Liniger, Phys. Rev. 130, 1605 (1963).

${ }^{63}$ G. G. Batrouni, R. T. Scalettar, and G. T. Zimanyi, Phys. Rev. Lett. 65, 1765 (1990).

${ }^{64}$ W. Kohn, Phys. Rev. 133, A171 (1964).

${ }^{65}$ D. G. Shelton, A. A. Nersesyan, and A. M. Tsvelik Phys. Rev. B 538521 (1996). 